\title{
Dinuclear copper(II) complexes of four new pyrazole-containing macrocyclic ligands are active catalysts in the oxidative coupling of 2,6-dimethylphenol
}

\author{
Anna M. Schuitema ${ }^{a}$, Peter G. Aubel ${ }^{a}$, Iryna A. Koval ${ }^{\mathrm{a}}$, Mireille Engelen ${ }^{\mathrm{a}}$, \\ Willem L. Driessen ${ }^{a, *}$, Jan Reedijk ${ }^{\mathrm{a}, *}$, Martin Lutz ${ }^{\mathrm{b}}$, Anthony L. Spek ${ }^{\mathrm{b}}$ \\ ${ }^{a}$ Leiden Institute of Chemistry, Gorlaeus Laboraties, Leiden University, P.O. Box 9502, 2300 RA Leiden, The Netherlands \\ ${ }^{\mathrm{b}}$ Bijvoet Centre for Biomolecular Research, Crystal and Structural Chemistry, Utrecht University, Padualaan 8, 3584 CH Utrecht, The Netherlands
}

Received 2 May 2003; accepted 24 May 2003

\begin{abstract}
Four new macrocyclic ligands of varying ring size, [22]py4pz, [22]pr4pz, [18]py2pz and [20]py2pz, containing four or two endocyclic pyrazole groups, form dinuclear copper compounds. The single crystal X-ray structures of $\left[\mathrm{Cu}(\mathrm{pr} 2 \mathrm{pz})(\mathrm{ClO})_{2}\right](\mathrm{A})$, $\left[\mathrm{Cu}_{2}([22] \mathrm{pr} 4 \mathrm{pz}) \mathrm{Cl}_{4}\right](\mathrm{MeOH})(\mathbf{B}),\left[\mathrm{Cu}_{4}([18] \mathrm{py} 2 \mathrm{pz})_{2}(\mu-\mathrm{Cl})_{4} \mathrm{Cl}_{2}\right] \mathrm{Cl}_{2}(\mathbf{C})$ and $\left[\mathrm{Cu}_{2}([18] \mathrm{py} 2 \mathrm{pz})\left(\mathrm{CH}_{3} \mathrm{CN}\right)_{4}\left(\mathrm{ClO}_{4}\right)_{2}\right]\left(\mathrm{ClO}_{4}\right)_{2}\left(\mathrm{CH}_{3} \mathrm{CN}\right)_{1.6}(\mathbf{D})$, show the pyrazole groups and the amine nitrogens all to be involved in the coordination of the copper(II) ions. In the mononuclear compound $\mathbf{A}$ the copper ion is in a distorted octahedral geometry, with the equatorial plane formed by four nitrogen donor atoms from the ligand and the axial positions occupied by two oxygen atoms from mono-coordinated perchlorate anions. In compound $\mathbf{B}$ each copper ion is in a distorted square pyramidal environment, with the three ligand nitrogens and a chloride atom in the equatorial plane and another chloride atom in the axial position. The cation of compound $\mathbf{C}$ contains four copper centers. Two bridging $\mathrm{Cl}$ atoms connect the two central copper atoms to form a centrosymmetric four membered ring. Two macrocyclic units are present in the cation, each containing two copper atoms bridged by a chloride atom. One copper is fivefold coordinated and the second copper is distorted octahedral. In compound $\mathbf{D}$ both copper(II) ions are in a distorted octahedral $\mathrm{N}_{5} \mathrm{O}$ environment, with the equatorial plane formed by the three ligand nitrogens and a nitrogen from an acetonitrile molecule. A second acetonitrile molecule and a mono-coordinated perchlorate anion are weakly bound in the axial positions. The copper nitrate compounds of these new ligands are to some degree active catalysts in the oxidative coupling of 2,6-dimethylphenol with molecular dioxygen to poly(2,6-dimethyl1,4-phenylene ether).
\end{abstract}

(C) 2003 Elsevier B.V. All rights reserved.

Keywords: Macrocycle; Dinuclear copper complexes; Crystal structures; Dimethyl phenol; Oxidative coupling

\section{Introduction}

Copper proteins with a type-3 site, like hemocyanin and the enzymes tyrosinase and catechol oxidase are important dioxygen-processing proteins [1-6]. These metallo-enzymes, which use molecular oxygen from air as the primary oxidant, are generally considered as very sophisticated catalysts. Their reactions are very fast and highly efficient while operating under mild conditions

* Corresponding authors. Tel.: +31-71-527 4450; fax: +31-71-527 4671.

E-mail address: reedijk@chem.leidenuniv.nl (J. Reedijk). with high substrate specificity, regio-selectivity and yield, and having mostly water as the only 'waste' product [7]. Because dioxygen-processing also plays an important role in industrial processes, the design of model compounds for type-3 site proteins has not only been driven by the significance of oxygen binding and activation in natural systems [8-13], but also by the prospect of using these compounds as industrial oxygenation catalysts [14-16].

The type- 3 site contains a pair of copper atoms each coordinated to three histidine residues from the protein backbone. In the oxy form of hemocyanin dioxygen was found to bridge between both copper ions in a $\mu-\eta^{2}: \eta^{2}$ fashion [17]. Tyrosinase and catechol oxidase have 
similar type-3 sites [2,6]. Our strategy in developing model compounds for the type- 3 site is to approach the architecture of the copper site, while keeping the entropy of self-assembly to a minimum. This strategy has resulted in the design and synthesis of new macrocyclic ligands with built-in pyrazole groups able to hold two copper ions in close proximity (Fig. 1). [22]py4pz differs from the previously reported macrocycle mepy $22 \mathrm{pz}$ $[18,19]$ in that it has no methyl substituents on the pyrazole rings.

The dinuclear copper(I) complex of $\left[\mathrm{Cu}_{2}([22] \mathrm{py} 4 \mathrm{pz})\right](\mathrm{X})_{2}$ reacts with dioxygen in the same way as the copper(I) complex of mepy22pz [18] to yield the peroxodicopper(II) complex $\left[\mathrm{Cu}_{2}([22]\right.$ py4p$\left.\mathrm{z})\left(\mathrm{O}_{2}\right)\right](\mathrm{X})_{2}\left(\mathrm{X}=\mathrm{ClO}_{4}, \mathrm{CF}_{3} \mathrm{SO}_{3}\right)$. These purple peroxo complexes are stable as powders at room temperature in air. Electronic spectra indicate a trans- $\mu-1,2$-binding mode for the peroxo anion. EXAFS and resonance Raman spectroscopy of the peroxo complex of mepy $22 \mathrm{pz}$ have confirmed the presence of the peroxide ion in the solid state [18]. The trans $-\mu-1,2$ binding mode of the peroxo may be the consequence of the $2 \times 4$-nitrogen donor surroundings, furnished by [22]py4pz and mepy22pz to the copper ions.

In an attempt to mimic the dinuclear type- 3 site more closely, macrocyclic ligands with a $2 \times 3$-nitrogen donor set were synthesized. [22]pr4pz lacks the exocyclic pyridine groups of [22]py4pz, whereas in [18]py2pz and [20]py2pz one bispyrazole side of [22]py4pz is replaced by an alkyl chain, (hexyl in the case of [18]py2pz and octyl in the case of [20]py2pz).

To test the capability to process dioxygen, the dicopper nitrate complexes of these new ligands have been used as precursor-catalysts in the oxidative cou- pling of 2,6-dimethylphenol (DMP). This copper-catalyzed polymerization leads to poly(2,6-dimethyl-1,4phenylene ether) (PPE), an amorphous industrial highperformance thermoplastic, and undesirable 4-(3,5-dimethyl-4-oxo-2,5-cyclohexadienylidene)-2,6-dimethyl2,5-cyclohexadienone (DPQ) [20,21] (Fig. 2). In most recent proposals the catalytically active intermediate in this oxidative coupling is believed to be a dinuclear ( $\mu$ hydroxo)( $\mu$-phenoxo) dicopper(II) species [22-27]. This intermediate is quite similar to the oxy form of the type3 site and it can be expected that the use of dinucleating ligands will lead to enhanced catalytic activity due to the ease of formation of dinuclear species in solution.

\section{Experimental}

\subsection{Materials and methods}

Most of the synthetic work was carried out using standard Schlenk techniques. All chemicals used were

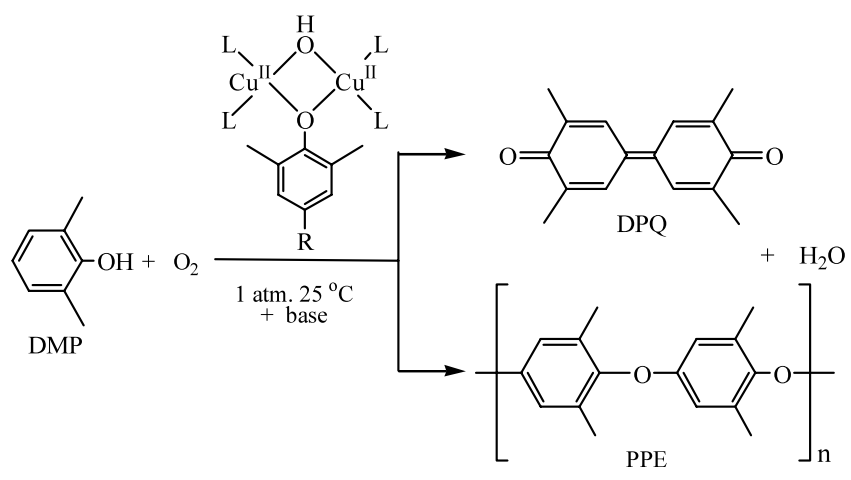

Fig. 2. The oxidative coupling of DMP to PPE and DPQ.
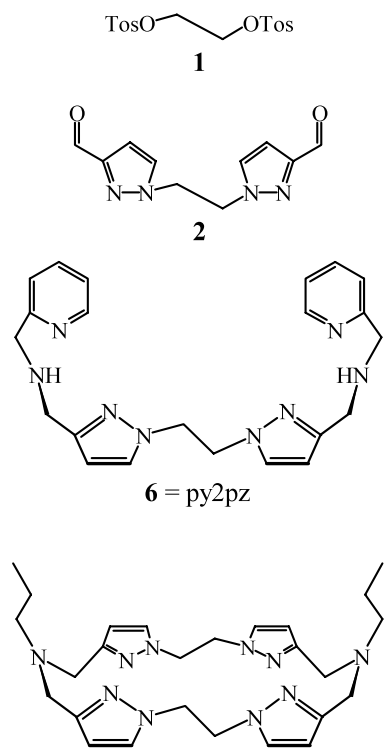

[22]pr4pz

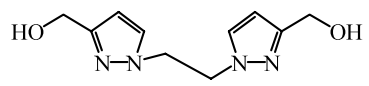

3

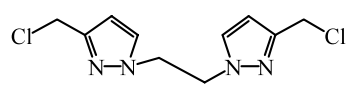

4

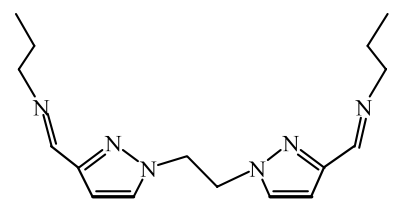

7

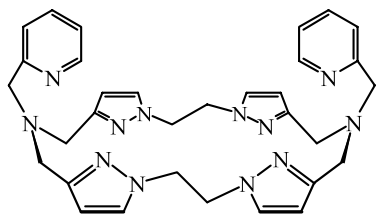

[22]py4pz
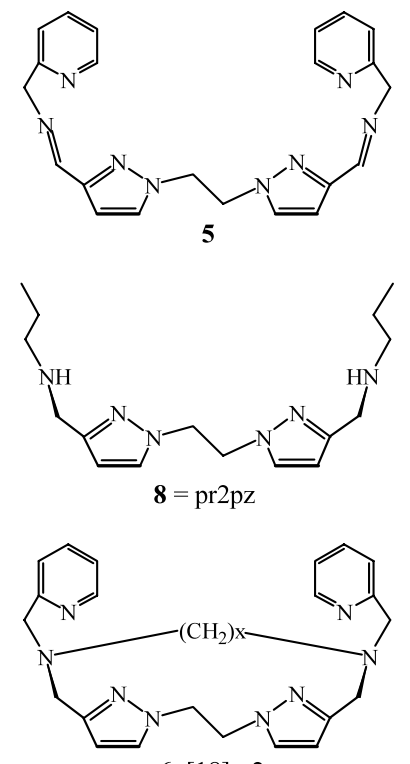

$\mathrm{x}=6:[18] \mathrm{pr} 2 \mathrm{pz}$
$\mathrm{x}=8:[20] \mathrm{pr} 2 \mathrm{pz}$

Fig. 1. The four new macrocycles and their precursors. 
commercially available and used without further purification unless stated otherwise. 3-Pyrazolyl aldehyde was purchased from Merck, 2-(aminomethyl)pyridine and tosyl chloride (tosCl) were obtained from Acros. Tetrahydrofuran (thf) was dried by reflux over sodium. DMP was recrystallized from $n$-hexane. Water was demineralized prior to use. NMR spectra were recorded on a JEOL FX-200 (200 MHz) FT-NMR spectrometer. Ligand field spectra of the solids $(300-2000 \mathrm{~nm}$, diffuse reflectance) were taken on a Perkin-Elmer 330 spectrophotometer equipped with a data station, with $\mathrm{MgO}$ as a reference. IR spectra were recorded as pure solid on a Perkin-Elmer FT-IR Paragon 1000 spectrophotometer with a Specac single-reflection diamond ATR P/N 10500. X-band electron paramagnetic resonance (EPR) measurements were performed at room temperature and at $77 \mathrm{~K}$ in the solid state, or at $77 \mathrm{~K}$ as methanol frozen solutions on a JEOL RE2x electron spin resonance spectrometer, using DPPH $(g=2.0036)$ as a standard. Dioxygen-uptake experiments were performed using an in-house designed apparatus, described in detail in earlier publications $[24,28]$.

Caution: Perchlorate salts of metal complexes with organic ligands are potentially explosive. Only small amounts of material should be prepared and handled with care.

\subsection{Synthesis of the macrocycles and their precursors}

Unless stated otherwise, the compounds were synthesized according to the procedures published by Bode [19] for the methylated variant of [22]py4pz (mepy22pz) and precursors. The slightly altered synthetic method with 3pyrazolylaldehyde as the starting compound instead of 5-methyl-3-pyrazolylaldehyde resulted in faster synthesis and higher yields (Fig. 1).

1,2-bis-Tosylate-ethane (1), was synthesized by the procedure described by Keegstra [29].

1,2-di(3'-Formylpyrazol-1'-pyrazolyl)ethane synthesized from 3-pyrazolylaldehyde and 1, was obtained as a slightly yellow colored powder. Yield: $62 \%$. ${ }^{1} \mathrm{H}$ NMR $\left(\mathrm{CDCl}_{3}-\mathrm{d}_{6}, 200 \mathrm{MHz}\right): \delta=9.97$ (s, $2 \mathrm{H}$, $\mathrm{C}(\mathrm{O}) H) ; 7.08\left(\mathrm{~d}, 2 \mathrm{H}, 4^{\prime} \mathrm{pz}-H,{ }^{3} J_{\mathrm{H}-\mathrm{H}}=2.3 \mathrm{~Hz}\right) ; 6.69$ (d, $\left.2 \mathrm{H}, 5^{\prime} \mathrm{pz}-\mathrm{H},{ }^{3} J_{\mathrm{H}-\mathrm{H}}=2.3 \mathrm{~Hz}\right) ; 4.73(\mathrm{~s}, 4 \mathrm{H}, \mathrm{pz}-$ $\mathrm{CH}_{2} \mathrm{CH}_{2}-$ pz). Elemental analysis (\%) for $\mathrm{C}_{10} \mathrm{H}_{10} \mathrm{~N}_{4} \mathrm{O}_{2}$ Found: (Calc.): C 54.6 (55.0); H 4.9 (4.6); N 25.7 (25.7). MS (ESI): $m / z 219\left[\mathrm{M}+\mathrm{H}^{+}\right]$.

1,2-di(3'-Hydroxymethyl-1'-pyrazolyl)ethane (3), resulted from the reduction of 2 . The resulting yellow solid still contained some salt, which however, did not effect the next step (halogenation with $\mathrm{SOCl}_{2}$ ). Yield: $>100 \%$. ${ }^{1} \mathrm{H}$ NMR $\left(\mathrm{CH}_{3} \mathrm{OH}-\mathrm{d}_{6}, 200 \mathrm{MHz}\right): \delta=7.13(\mathrm{~d}, 2 \mathrm{H}$, $\left.4^{\prime} \mathrm{pz}-H,{ }^{3} J_{\mathrm{H}-\mathrm{H}}=2.3 \mathrm{~Hz}\right) ; 6.16\left(\mathrm{~d}, 2 \mathrm{H}, 5^{\prime} \mathrm{pz}-H,{ }^{3} J_{\mathrm{H}-\mathrm{H}}=\right.$ $2.3 \mathrm{~Hz}) ; 4.54$ (s, 4H, pz-CH $-\mathrm{OH}) ; 4.48(\mathrm{~s}, 4 \mathrm{H}, \mathrm{pz}-$ $\left.\mathrm{CH}_{2} \mathrm{CH}_{2}-\mathrm{pz}\right)$.
1,2-di(3'-Chloromethyl-1'-pyrazolyl)ethane (4), prepared from 3 by halogenation with $\mathrm{SOCl}_{2}$, was obtained as an off white shiny solid. Yield: (relative to 2): $64 \%$. ${ }^{1} \mathrm{H}$ $\operatorname{NMR}\left(\mathrm{CDCl}_{3}-\mathrm{d}_{6}, 200 \mathrm{MHz}\right): \delta=6.91\left(\mathrm{~d}, 2 \mathrm{H}, 4^{\prime} \mathrm{pz}-H\right.$, $\left.{ }^{3} J_{\mathrm{H}-\mathrm{H}}=2.3 \mathrm{~Hz}\right) ; 6.17\left(\mathrm{~d}, 2 \mathrm{H}, 5^{\prime} \mathrm{pz}-H,{ }^{3} J_{\mathrm{H}-\mathrm{H}}=2.3 \mathrm{~Hz}\right)$; 4.59 (s, $4 \mathrm{H}, \mathrm{pz}-\mathrm{CH}_{2}-\mathrm{Cl}$ ); 4.51 (s, $4 \mathrm{H}, \mathrm{pz}-\mathrm{CH}_{2} \mathrm{CH}_{2}-\mathrm{pz}$ ). Elemental analysis (\%) for $\mathrm{C}_{10} \mathrm{H}_{12} \mathrm{~N}_{4} \mathrm{Cl}_{2}$ Found: (Calc.): C 45.8 (46.4); H 5.3 (4.7); N 21.3 (21.6). MS (ESI): $\mathrm{m} / \mathrm{z}$ $260\left[\mathrm{M}+\mathrm{H}^{+}\right]$.

1,2-di(3'-(2-Pyridin-2-ylmethylimino)-1'-pyrazoly1)ethane (5), prepared by the condensation of 2 with 2 (aminomethyl)pyridine, was obtained as a clear yellow oil. ${ }^{1} \mathrm{H}$ NMR $\left(\mathrm{CDCl}_{3}-\mathrm{d}_{6}, 200 \mathrm{MHz}\right): \delta=8.53(\mathrm{~d}, 2 \mathrm{H}$, $\left.6^{\prime} \mathrm{py}-H\right) ; 8.45\left(\mathrm{~s}, 2 \mathrm{H}, 3{ }^{\prime} \mathrm{pz}-\mathrm{CH}=\mathrm{N}\right) ; 7.63(\mathrm{dd}, 2 \mathrm{H}$, 4'py $-H) ; 7.38$ (d, 2H, 3'py $-H) ; 7.16\left(\mathrm{~m}, 2 \mathrm{H}, 5^{\prime}\right.$ py $\left.-H\right)$; $6.93\left(\mathrm{~d}, 2 \mathrm{H}, 5^{\prime} \mathrm{pz}-H\right) ; 6.59\left(\mathrm{~d}, 2 \mathrm{H}, 4^{\prime} \mathrm{pz}-H\right) ; 4.90(\mathrm{~s}, 4 \mathrm{H}$, py $\left.-\mathrm{CH}_{2}-\mathrm{N}\right) ; 4.56\left(\mathrm{~s}, 4 \mathrm{H}, \mathrm{pz}-\mathrm{CH}_{2}-\mathrm{CH}_{2}-\mathrm{pz}\right)$.

1,2-di-(3'-(2-Pyridin-2-ylmethylamino)-1'-pyrazoly1)ethane $(\mathbf{6}, \mathrm{py} 2 \mathrm{pz})$ resulted from the reduction of $\mathbf{5}$. The product was obtained as a clear yellow viscous oil. Yield starting from 2: 95\%. ${ }^{1} \mathrm{H}$ NMR $\left(\mathrm{CDCl}_{3}-\mathrm{d}_{6}, 200 \mathrm{MHz}\right)$ : $\delta=8.53\left(\mathrm{~d}, 2 \mathrm{H}, 6^{\prime} \mathrm{py}-H\right) ; 7.63\left(\mathrm{dd}, 2 \mathrm{H}, 4^{\prime} \mathrm{py}-H\right) ; 7.30$ $\left(\mathrm{d}, 2 \mathrm{H}, 3^{\prime} \mathrm{py}-H\right) ; 7.14\left(\mathrm{~m}, 2 \mathrm{H}, 5^{\prime} \mathrm{py}-H\right) ; 6.91(\mathrm{~d}, 2 \mathrm{H}$, $\left.5^{\prime} \mathrm{pz}-H,{ }^{3} J_{\mathrm{H}-\mathrm{H}}=2.3 \mathrm{~Hz}\right) ; 6.09\left(\mathrm{~d}, 2 \mathrm{H}, 4^{\prime} \mathrm{pz}-H,{ }^{3} J_{\mathrm{H}-\mathrm{H}}=\right.$ $2.3 \mathrm{~Hz}$ ); 4.48 (s, 4H, pz-CH $\left.-\mathrm{CH}_{2}-\mathrm{pz}\right) ; 3.95$ (s, 4H, py $\left.-\mathrm{CH}_{2}-\mathrm{N}\right) ; 3.86(\mathrm{~s}, 4 \mathrm{H}$, pz-CH $-\mathrm{C}-\mathrm{N})$. Elemental analysis (\%) for $\mathrm{C}_{22} \mathrm{H}_{26} \mathrm{~N}_{8}$ Found: (Calc.): $\mathrm{C} 63.6$ (65.7); H 4.8 (6.5); N 27.2 (27.8). MS (ESI): $\mathrm{m} / \mathrm{z} 403$ $\left[\mathrm{M}+\mathrm{H}^{+}\right] ; 425\left[\mathrm{M}+\mathrm{Na}^{+}\right]$.

1,2-di(3'-2-Propylimino)-1'-pyrazolyl)ethane (7) was prepared by the condensation of $\mathbf{2}$ with propylamine. In situ the reduction to amino $\mathbf{8}$ is done.

1,2-di(3'-2-Propyl-2-ylmethylamino)-5'-methyl-1'pyrazolyl)-ethane $(\mathbf{8}, \mathrm{pr} 2 \mathrm{pz})$ resulted from the reduction of 7. The product was obtained as a light yellow oil that crystallizes on standing. Yield (starting from 2): $94 \% .{ }^{1} \mathrm{H}$ NMR $\left(\mathrm{CDCl}_{3}-\mathrm{d}_{6}, 200 \mathrm{MHz}\right): 6.88$ (d, 4H, 5' pz-H, $\left.{ }^{3} J_{\mathrm{H}-\mathrm{H}}=2.1 \mathrm{~Hz}\right) ; 6.05\left(\mathrm{~d}, 4 \mathrm{H}, 4^{\prime} \mathrm{pz}-H,{ }^{3} J_{\mathrm{H}-\mathrm{H}}=2.1 \mathrm{~Hz}\right)$; 4.47 (s, $\left.4 \mathrm{H}, \mathrm{pz}-\mathrm{CH}_{2}-\mathrm{CH}_{2}-\mathrm{pz}\right) ; 3.79$ (s, $4 \mathrm{H}, \mathrm{pz}-\mathrm{CH}_{2}-$ $\mathrm{N}) ; 2.62\left(\mathrm{t}, 4 \mathrm{H}, \mathrm{N}-\mathrm{CH}_{2}-\mathrm{CH}_{2}-\mathrm{CH}_{3},{ }^{3} J_{\mathrm{H}-\mathrm{H}}=7.2 \mathrm{~Hz}\right)$; $1.56\left(\mathrm{~m}, 4 \mathrm{H}, \mathrm{N}-\mathrm{CH}_{2}-\mathrm{CH}_{2}-\mathrm{CH}_{3}, 2 \times{ }^{3} J_{\mathrm{H}-\mathrm{H}}=7.2 \mathrm{~Hz}\right)$; $0.92\left(\mathrm{t}, 6 \mathrm{H}, \mathrm{N}-\mathrm{CH}_{2}-\mathrm{CH}_{2}-\mathrm{CH}_{3},{ }^{3} \mathrm{~J}_{\mathrm{H}-\mathrm{H}}=7.2 \mathrm{~Hz}\right)$. Elemental analysis (\%) for $\mathrm{C}_{16} \mathrm{H}_{28} \mathrm{~N}_{6}$ Found: (Calc.): $\mathrm{C} 61.4$ (63.1); H 8.0 (9.3); N 27.2 (27.6). MS (ESI): $m / z 305$ $\left[\mathrm{M}+\mathrm{H}^{+}\right]$.

9,22-bis(Pyridin-2'-ylmethyl)$1,4,9,14,17,22,27,28,29,30$-decaazapentacyclo[22.2.1.14,7.111,14.117,20]triacontane$5,7(28), 11(29), 12,18,20(30), 24(27), 25$-octaene ([22]py4pz) synthesized from $\mathbf{4}$ and $\mathbf{6}$, was obtained as a brown solid. The solid was recrystallized without purification by column chromatography. The solid was dissolved in warm thf, after which hexane and diethyl ether were added (thf:hexane:diethyl ether, 1:1:1). Cooling to $255 \mathrm{~K}$ yielded white (crystalline) solid. Yield: $21 \%$. ${ }^{1} \mathrm{H}$ NMR (MeOH-d $\left.6,200 \mathrm{MHz}\right): 8.40$ (d, 2H, 6' py-H); 
$7.81\left(\mathrm{dt}, 2 \mathrm{H}, 4^{\prime}\right.$ py $\left.-H\right) ; 7.64\left(\mathrm{~d}, 2 \mathrm{H}, 3^{\prime} \mathrm{py}-H\right) ; 7.41(\mathrm{~d}$, $\left.4 \mathrm{H}, 5^{\prime} \mathrm{pz}-H,{ }^{3} J_{\mathrm{H}-\mathrm{H}}=2.1 \mathrm{~Hz}\right): 7.25\left(\mathrm{~m}, 4 \mathrm{H}, 5^{\prime} \mathrm{py}-H\right)$; $6.11\left(\mathrm{~d}, 4 \mathrm{H}, 4^{\prime} \mathrm{pz}-H,{ }^{3} J_{\mathrm{H}-\mathrm{H}}=2.6 \mathrm{~Hz}\right) ; 4.52(\mathrm{~s}, 8 \mathrm{H}, \mathrm{pz}-$ $\left.\mathrm{CH}_{2}-\mathrm{CH}_{2}-\mathrm{pz}\right) ; 3.56\left(\mathrm{~s}, 4 \mathrm{H}, \mathrm{py}-\mathrm{CH}_{2}-\mathrm{N}\right) ; 3.36(\mathrm{~s}, 8 \mathrm{H}$, $\left.\mathrm{pz}-\mathrm{CH}_{2}-\mathrm{N}\right)$. Elemental analysis $(\%)$ for $\mathrm{C}_{32} \mathrm{H}_{36} \mathrm{~N}_{12}$ Found: (calc.): C 65.4 (65.3); H 6.3 (6.2); N 28.8 (28.6). MS (ESI): $m / z 589\left[\mathrm{M}+\mathrm{H}^{+}\right]: 611\left[\mathrm{M}+\mathrm{Na}^{+}\right]$: $627\left[\mathrm{M}+\mathrm{K}^{+}\right]$.

9,22-Dipropyl-1,4,9,14,17,22,27,28,29,30-decaazapentacyclo[22.2.1.14,7.111,14.117,20]triacontane-

5,7(28),11(29),12,18,20(30),24(27),25-octaene

([22]pr4pz), was synthesized from $\mathbf{4}$ and $\mathbf{8}$ according to the same synthetic route as for [22]py4pz. But instead of 1 equiv. of $\mathbf{6}, 1$ equiv. of $\mathbf{8}$ was added. The crude product was obtained as a light yellow powder. White crystals were obtained by crystallization from methanol/diethyl ether. Yield: $22 \% .{ }^{1} \mathrm{H}$ NMR $\left(\mathrm{CDCl}_{3}-\mathrm{d}_{6}, 200 \mathrm{MHz}\right): 7.06$ (d, $\left.4 \mathrm{H}, 5^{\prime} \mathrm{pz}-H,{ }^{3} J_{\mathrm{H}-\mathrm{H}}=2.1 \mathrm{~Hz}\right) ; 6.02\left(\mathrm{~d}, 4 \mathrm{H}, 4^{\prime} \mathrm{pz}-H\right.$, $\left.{ }^{3} J_{\mathrm{H}-\mathrm{H}}=2.1 \mathrm{~Hz}\right) ; 4.49\left(\mathrm{~s}, 8 \mathrm{H}, \mathrm{pz}-\mathrm{CH}_{2}-\mathrm{CH}_{2}-\mathrm{pz}\right) ; 3.43$ (s, $\left.8 \mathrm{H}, \mathrm{pz}-\mathrm{CH}_{2}-\mathrm{N}\right) ; 2.31\left(\mathrm{t}, 4 \mathrm{H}, \mathrm{N}-\mathrm{CH}_{2}-\mathrm{CH}_{2}-\mathrm{CH}_{3}\right.$, $\left.{ }^{3} J_{\mathrm{H}-\mathrm{H}}=7.4 \mathrm{~Hz}\right) ; 1.55\left(\mathrm{~m}, 4 \mathrm{H}, \mathrm{N}-\mathrm{CH}_{2}-\mathrm{CH}_{2}-\mathrm{CH}_{3}\right.$, $\left.2 \times{ }^{3} J_{\mathrm{H}-\mathrm{H}}=7.4 \mathrm{~Hz}\right) ; 0.85\left(\mathrm{t}, 6 \mathrm{H}, \mathrm{N}-\mathrm{CH}_{2}-\mathrm{CH}_{2}-\mathrm{CH}_{3}\right.$, ${ }^{3} J_{\mathrm{H}-\mathrm{H}}=7.4 \mathrm{~Hz}$ ). Elemental analysis (\%) for $\mathrm{C}_{26} \mathrm{H}_{38} \mathrm{~N}_{10}$ Found: (Calc.): C 63.5 (63.7); H 8.1 (7.8); N 28.9 (28.6). MS (ESI): $m / z 491\left[\mathrm{M}+\mathrm{H}^{+}\right]: 513\left[\mathrm{M}+\mathrm{Na}^{+}\right]$.

9,16-bis(Pyridin-2'-ylmethyl)-1,4,9,16,21,22-hexaazatricyclo-[16.2.1.14,7]docosane-5,7(22),18(21),19-tetraene ([18]py2pz) was synthesized from 6 and 1,6-dibromohexane according to the same synthetic route as for [22]py4pz. But instead of 1 equiv. of 4,1 equiv. of dibromohexane was added. The crude product was obtained as a red-brown oil. Addition of acetonitrile and concentration of the dark-yellow solution resulted in precipitation of white crystals, which were filtered and washed several times with diethyl ether. Yield: $10 \% .{ }^{1} \mathrm{H}$ NMR $\left(\mathrm{MeOH}-\mathrm{d}_{4}, 300 \mathrm{MHz}\right): 8.44$ (d, 2H, 6' py $-H$, $\left.{ }^{3} J_{\mathrm{H}-\mathrm{H}}=3.3 \mathrm{~Hz}\right) ; 7.83\left(\mathrm{dd}, 2 \mathrm{H}, 4^{\prime} \mathrm{py}-H,{ }^{3} J_{\mathrm{H}-\mathrm{H}}=5.2 \mathrm{~Hz}\right.$ and $5.0 \mathrm{~Hz}) ; 7.68\left(\mathrm{~d}, 2 \mathrm{H}, 3^{\prime}\right.$ py $\left.-H,{ }^{3} J_{\mathrm{H}-\mathrm{H}}=5.2 \mathrm{~Hz}\right) ; 7.38$ $\left(\mathrm{d}, 2 \mathrm{H}, 5^{\prime} \mathrm{pz}-H,{ }^{3} J_{\mathrm{H}-\mathrm{H}}=1.5 \mathrm{~Hz}\right) ; 7.30\left(\mathrm{~m}, 2 \mathrm{H}, 5^{\prime} \mathrm{py}-H\right.$, ${ }^{3} J_{\mathrm{H}-\mathrm{H}}=3.3 \mathrm{~Hz}$ and $\left.5.0 \mathrm{~Hz}\right) ; 6.14\left(\mathrm{~d}, 2 \mathrm{H}, 4^{\prime} \mathrm{pz}-H\right.$, $\left.{ }^{3} J_{\mathrm{H}-\mathrm{H}}=1.5 \mathrm{~Hz}\right) ; 4.55\left(\mathrm{~s}, 4 \mathrm{H}, \mathrm{pz}-\mathrm{CH}_{2}-\mathrm{CH}_{2}-\mathrm{pz}\right) ; 3.76$ (s, $\left.4 \mathrm{H}, \mathrm{py}-\mathrm{CH}_{2}-\mathrm{N}\right) ; 3.45\left(\mathrm{~s}, 4 \mathrm{H}, \mathrm{pz}-\mathrm{CH}_{2}-\mathrm{N}\right) ; 2.31(\mathrm{t}$, $\left.4 \mathrm{H}, \mathrm{N}-\mathrm{CH}_{2}-\mathrm{CH}_{2}-\mathrm{CH}_{2},{ }^{3} \mathrm{~J}_{\mathrm{H}-\mathrm{H}}=4.3 \mathrm{~Hz}\right) ; 1.37(\mathrm{~m}, 4 \mathrm{H}$, $\left.\mathrm{N}-\mathrm{CH}_{2}-\mathrm{CH}_{2}-\mathrm{CH}_{2}\right) ; 1.10\left(\mathrm{~m}, 4 \mathrm{H}, \quad \mathrm{N}-\mathrm{CH}_{2}-\mathrm{CH}_{2}-\right.$ $\mathrm{CH}_{2}$ ). Elemental analysis (\%) for $\mathrm{C}_{28} \mathrm{H}_{36} \mathrm{~N}_{8}$ Found: (Calc.): C 68.5 (69.4); H 7.3 (7.5); N 22.9 (23.1). MS (ESI): $m / z 485\left[\mathrm{M}+\mathrm{H}^{+}\right]$.

9,18-bis(Pyridin-2'-ylmethyl)-1,4,9,18,23,24-hexaazatricyclo-[18.2.1.14,7]tetracosane-5,7(24),20(23),21-tetraene ([20]py2pz) was synthesized from $\mathbf{6}$ and $1,8-$ dibromooctane according to the same synthetic route as for [22]py4pz. But instead of $\mathbf{4}$, dibromooctane was added. The crude product was obtained as a red-brown oil. Addition of acetonitrile and concentration of the dark-yellow solution resulted in precipitation of white crystals, which were filtered and washed several times with diethyl ether. Yield: $25.2 \% .{ }^{1} \mathrm{H}$ NMR $\left(\mathrm{MeOH}-\mathrm{d}_{4}\right.$, $300 \mathrm{MHz}): 8.44$ (d, 2H, $\left.6^{\prime} \mathrm{py}-H,{ }^{3} J_{\mathrm{H}-\mathrm{H}}=3.6 \mathrm{~Hz}\right) ; 7.82$ (dd, 2H, 4'py-H); 7.67 (m, 2H, 3'py-H); $7.30(\mathrm{~m}, 2 \mathrm{H}$, $\left.5^{\prime} \mathrm{py}-H\right) ; 7.23\left(\mathrm{~d}, 2 \mathrm{H}, 5^{\prime} \mathrm{pz}-H,{ }^{3} J_{\mathrm{H}-\mathrm{H}}=2.1 \mathrm{~Hz}\right) ; 6.15(\mathrm{~d}$, $\left.2 \mathrm{H}, 4^{\prime} \mathrm{pz}-H,{ }^{3} J_{\mathrm{H}-\mathrm{H}}=2.1 \mathrm{~Hz}\right) ; 4.50\left(\mathrm{~s}, 4 \mathrm{H}, \mathrm{pz}-\mathrm{CH}_{2}-\right.$ $\left.\mathrm{CH}_{2}-\mathrm{pz}\right) ; 3.80\left(\mathrm{~s}, 4 \mathrm{H}, \mathrm{py}-\mathrm{CH}_{2}-\mathrm{N}\right) ; 3.56(\mathrm{~s}, 4 \mathrm{H}, \mathrm{pz}-$ $\left.\mathrm{CH}_{2}-\mathrm{N}\right) ; 2.38 \quad\left(\mathrm{t}, \quad 4 \mathrm{H}, \quad \mathrm{N}-\mathrm{CH}_{2}-\mathrm{CH}_{2}-\mathrm{CH}_{2}-\mathrm{CH}_{2}\right.$, $\left.{ }^{3} J_{\mathrm{H}-\mathrm{H}}=3.6 \mathrm{~Hz}\right) ; 1.36\left(\mathrm{~m}, 4 \mathrm{H}, \mathrm{N}-\mathrm{CH}_{2}-\mathrm{CH}_{2}-\mathrm{CH}_{2}-\right.$ $\left.\mathrm{CH}_{2}\right) ; 1.21\left(\mathrm{~m}, 8 \mathrm{H}, \mathrm{N}-\mathrm{CH}_{2}-\mathrm{CH}_{2}-\mathrm{CH}_{2}-\mathrm{CH}_{2}\right.$ ). Elemental analysis (\%) for $\mathrm{C}_{30} \mathrm{H}_{40} \mathrm{~N}_{8}$ Found: (Calc.): $\mathrm{C} 70.0$ (70.3); H 8.5 (7.9); N 22.1 (21.9). MS (ESI): $\mathrm{m} / z 514$ $\left[\mathrm{M}+\mathrm{H}^{+}\right]: 635\left[\mathrm{M}+\mathrm{Na}^{+}\right]$.

\subsection{Synthesis of the coordination compounds}

Unless stated otherwise the coordination compounds were obtained by adding a solution of the ligand in methanol to a solution of 2 equiv. of copper salt in methanol. Single crystals were obtained by slow diffusion of diethyl ether into these solutions. In the case of $\left[\mathrm{Cu}(\mathrm{pr} 2 \mathrm{pz})\left(\mathrm{ClO}_{4}\right)_{2}\right] \quad$ (A) and $\left[\mathrm{Cu}_{4}([18] \mathrm{py} 2 \mathrm{pz})_{2}(\mu-\right.$ $\left.\mathrm{Cl})_{4} \mathrm{Cl}_{2}\right] \mathrm{Cl}_{2}$ (C) a methanol/acetonitrile mixture was used as solvent. In the case of $\left[\mathrm{Cu}_{2}([22] \mathrm{pr} 4 \mathrm{pz}) \mathrm{Cl}_{4}\right](\mathrm{MeOH})$ (B) an excess of ligand was used to prevent formation of crystals with other compositions. The single crystals of $\left[\mathrm{Cu}_{2}([18]\right.$ py2pz $\left.)\left(\mathrm{CH}_{3} \mathrm{CN}\right)_{4}\left(\mathrm{ClO}_{4}\right)_{2}\right]\left(\mathrm{ClO}_{4}\right)_{2}\left(\mathrm{CH}_{3} \mathrm{CN}\right)_{1.6}$ (D) were obtained by recrystallizing the blue solid, obtained from the methanol solution, in acetonitrile. The compounds were characterized by elemental analyses, IR, EPR and LF spectra. Spectroscopic data are listed in Table 1.

\section{4. $X$-ray structure determinations}

X-ray intensities were measured on a Nonius KappaCCD diffractometer with rotating anode $(\lambda=0.71073$ $\AA$ ) at a temperature of 150(2) K. The structures were solved with automated Patterson methods (DIRDIF-97 [30]) (compounds $\mathbf{A}, \mathbf{B}$, and $\mathbf{D}$ ) or direct methods (sHELXS-97 [31]) (compound C). The structure refinement was done with SHELXL-97 against $F^{2}$ of all reflections. Molecular illustrations, checking for higher symmetry, and geometry calculations were performed with the PLATON [32] package. The crystal structure of compound C contains large voids $\left(1929.1 \AA^{3}\right.$ unit cell $^{-1}$ ) filled with disordered solvent molecules. Their contribution to the structure factors was secured by back-Fourier transformation (program PLATON [32]CALC SQUEEZE, $303 \mathrm{e}^{-}$unit cell ${ }^{-1}$ ). For all structures a selection of the crystal data and additional parameters are presented in Table 2. Selected bond distances and bond angles are given in Tables 3-6. 
Table 1

Relevant spectroscopic and analytical data of $\left[\mathrm{Cu}(\mathrm{pr} 2 \mathrm{pz})\left(\mathrm{ClO}_{4}\right)_{2}\right](\mathbf{A}),\left[\mathrm{Cu}_{2}([22] \mathrm{pr} 4 \mathrm{pz}) \mathrm{Cl}_{4}\right]\left(\mathrm{CH}_{3} \mathrm{OH}\right)(\mathbf{B}), \quad\left[\mathrm{Cu}_{4}([18] \mathrm{py} 2 \mathrm{pz})_{2}\left(\mu-\mathrm{Cl}_{4} \mathrm{Cl}_{2}\right] \mathrm{Cl}_{2}(\mathbf{C})\right.$, $\left[\mathrm{Cu}_{2}([18] \mathrm{py} 2 \mathrm{pz})\left(\mathrm{CH}_{3} \mathrm{CN}\right)_{4}\left(\mathrm{ClO}_{4}\right)_{2}\right]\left(\mathrm{ClO}_{4}\right)_{2}\left(\mathrm{CH}_{3} \mathrm{CN}\right)_{1.6}(\mathrm{D})$

\begin{tabular}{lllll}
\hline \multicolumn{1}{c}{ Color } & Elemental analysis, \% Found: (Calc.) & Ligand field $\left(10^{3} \mathrm{~cm}^{-1}\right)$ & EPR solid at 295 and $77 \mathrm{~K}$ \\
\hline A & dark blue & C 33.5 (33.9); H 5.4 (5.0); N 14.7 (14.8) & 17.4 (in methanol: 16.4) & $g_{\perp}=2.06, g_{\|}=2.20, A_{\|}=19.4(\mathrm{mT})$ \\
B & light green & C 39.6 (41.0); H 5.3 (5.4); N 18.0 (17.7) & 11.6 (broad) & $g_{\perp}=2.05, g_{\|}=2.17$ \\
C & greenish blue & C 44.4 (44.6); H 4.7 (4.8); N 14.8 (14.9) & 12.4 (broad) & $g_{\perp}=2.03, g_{\|}=2.22$ \\
D dark blue & crystals deteriorate when separated from the mother liquid & 15.7 & $g_{\perp}=2.08$ \\
\hline
\end{tabular}

\subsection{Oxidative coupling reactions}

All oxidative coupling reactions were performed in acetonitrile. In all cases copper nitrate was used to maintain a certain level of consistency with the present and with previous oxidative coupling experiments $[24,28]$. In a typical quantitative, time-resolved oxidative coupling experiment, one compartment of a special twocompartment reaction vessel was filled with $10 \mathrm{ml}$ of a solution containing $0.025 \mathrm{mmol}$ ligand and $0.05 \mathrm{mmol}$ $\mathrm{Cu}\left(\mathrm{NO}_{3}\right)_{2}$. The other compartment was filled with $5 \mathrm{ml}$ of a $0.6 \mathrm{M}$ DMP solution and a small amount of a highly concentrated solution of $0.1 \mathrm{mmol}$ of $\mathrm{NaOMe}$ in methanol. Intense shaking in a dioxygen atmosphere starts the reaction, allowing the two reactant solutions to mix instantly and intimately with each other and with dioxygen. The resulting reaction mixture was $1.66 \mathrm{mM}$ in ligand, $3.33 \mathrm{mM}$ in copper, and 0.2 $\mathrm{M}$ in DMP. All polymerization reactions were performed at $25^{\circ} \mathrm{C}$ under pure dioxygen at atmospheric pressure. The catalytic activity has been determined by calculating the initial dioxygen-uptake rate $R_{0}$. After the experiment, the reaction mixture was diluted with acetonitrile and the amount of DPQ formed was measured spectrophotometrically. Since PPE and DPQ are virtually the sole products of the reaction, the amount of PPE formed can be calculated from the amount of dioxygen consumed, corrected for the amount of DPQ formed. A

Table 2

Crystallographic data for $\left[\mathrm{Cu}(\mathrm{pr} 2 \mathrm{pz})\left(\mathrm{ClO}_{4}\right)_{2}\right]$ $\left[\mathrm{Cu}_{2}([18] \mathrm{py} 2 \mathrm{pz})\left(\mathrm{CH}_{3} \mathrm{CN}\right)_{4}\left(\mathrm{ClO}_{4}\right)_{2}\right]\left(\mathrm{ClO}_{4}\right)_{2}\left(\mathrm{CH}_{3} \mathrm{CN}\right)_{1.6}(\mathbf{D})$

\begin{tabular}{|c|c|c|c|c|}
\hline & $\mathbf{A}$ & B & $\mathbf{C}$ & D \\
\hline Formula & $\mathrm{C}_{16} \mathrm{H}_{28} \mathrm{Cl}_{12} \mathrm{CuN}_{6} \mathrm{O}_{8}$ & $\mathrm{C}_{26} \mathrm{H}_{38} \mathrm{Cl}_{4} \mathrm{Cu}_{2} \mathrm{~N}_{10} \cdot \mathrm{CH}_{3} \mathrm{OH}$ & {$\left[\mathrm{C}_{28} \mathrm{H}_{36} \mathrm{Cl}_{3} \mathrm{Cu}_{2} \mathrm{~N}_{8}\right] \mathrm{Cl}^{\mathrm{a}}$} & $\mathrm{C}_{36} \mathrm{H}_{48} \mathrm{Cl}_{4} \mathrm{Cu}_{2} \mathrm{~N}_{12} \mathrm{O}_{16} \cdot 1.6 \mathrm{C}_{2} \mathrm{H}_{3} \mathrm{~N}$ \\
\hline Formula weight & 566.88 & 791.59 & $753.53^{\mathrm{a}}$ & 1239.43 \\
\hline Crystal system & monoclinic & monoclinic & orthorhombic & monoclinic \\
\hline Space group & $P 2_{1} / c$ (No. 14$)$ & $P 2_{1} / c$ (No. 14$)$ & $P b c a($ No. 61) & $P c$ (No. 7$)$ \\
\hline \multicolumn{5}{|l|}{ Unit cell dimensions } \\
\hline$a(\AA)$ & $12.0461(1)$ & $8.5078(1)$ & $13.5814(1)$ & $11.5374(2)$ \\
\hline$b(\AA)$ & $8.3670(1)$ & $14.2545(2)$ & $22.8088(2)$ & $10.2229(2)$ \\
\hline$c(\AA)$ & $23.9388(2)$ & $13.8566(2)$ & $25.1603(2)$ & $23.1116(5)$ \\
\hline$\beta\left(^{\circ}\right)$ & $104.9406(3)$ & $91.3677(5)$ & 90 & $95.7232(8)$ \\
\hline$V\left(\mathrm{~A}^{3}\right)$ & $2331.22(4)$ & $1679.97(4)$ & $7794.04(11)$ & $2712.33(9)$ \\
\hline $\mathrm{Z}$ & 4 & 2 & 8 & 2 \\
\hline$D_{\text {calc }}\left(\mathrm{g} \mathrm{cm}^{-3}\right)$ & 1.615 & 1.565 & $1.284^{\mathrm{a}}$ & 1.518 \\
\hline$F\left(\begin{array}{lll}0 & 0 & 0\end{array}\right)$ & 1172 & 816 & $3088^{\mathrm{a}}$ & 1274 \\
\hline$\mu\left(\mathrm{mm}^{-1}\right)$ & 1.22 & 1.62 & $1.39^{\mathrm{a}}$ & 1.06 \\
\hline Absorption correction & PLATON (MULABS) & PLATON (MULABS) & PLATON (ABST) & none \\
\hline Transmission range & $0.53-0.62$ & $0.78-0.85$ & $0.76-0.94$ & \\
\hline Crystal color & blue & light green & blue & blue \\
\hline Temperature (K) & 150 & 150 & 150 & 150 \\
\hline$\theta$ Min, $\max \left({ }^{\circ}\right)$ & $2.59,27.49$ & $2.39,27.47$ & $1.79,23.00$ & $1.77,25.50$ \\
\hline Total data collected & 31093 & 23936 & 64170 & 29098 \\
\hline Independent reflection $\left(R_{\text {int }}\right)$ & $5318(0.038)$ & $3835(0.048)$ & $5421(0.055)$ & $9827(0.050)$ \\
\hline Observed reflection $[I>2 \sigma(I)]$ & 4837 & 3325 & 4722 & 8576 \\
\hline Npar, Restraints & 328,123 & 207,0 & 379,6 & 723,272 \\
\hline$R_{1}[I>2 \sigma(I)], w R_{2}$ (all reflection), $\mathrm{S}$ & $0.0485,0.1463,1.06$ & $0.0311,0.0772,1.07$ & $0.0535,0.1243,1.13$ & $0.0381,0.0910,1.02$ \\
\hline Min and max resd. dens. $\left(\mathrm{e}^{-3}\right)$ & $-0.63,2.16$ & $-0.39,0.67$ & $-0.86,0.87$ & $-0.38,0.49$ \\
\hline
\end{tabular}

a The crystal structure contains disordered solvent molecules, which were handled as diffuse electron density (see Section 2). Derived values (Fw, $\left.D_{\text {calc }}, F\left(\begin{array}{lll}0 & 0 & 0\end{array}\right), \mu\right)$ do not contain the contribution of the disordered solvent. 
Table 3

Selected distances $(\AA)$ and angles $\left({ }^{\circ}\right)$ for $\left[\mathrm{Cu}(\mathrm{pr} 2 \mathrm{pz})\left(\mathrm{ClO}_{4}\right)_{2}\right](\mathbf{A})$

\begin{tabular}{|c|c|c|c|}
\hline \multicolumn{4}{|l|}{ Bond lengths } \\
\hline $\mathrm{Cu} 1-\mathrm{O} 51$ & $2.451(3)$ & $\mathrm{Cu} 1-\mathrm{N} 17$ & $2.044(3)$ \\
\hline $\mathrm{Cu} 1-061 \mathrm{a}^{\mathrm{a}}$ & $2.924(8)$ & $\mathrm{Cu} 1-\mathrm{N} 32$ & $1.971(3)$ \\
\hline $\mathrm{Cu} 1-\mathrm{O} 61 \mathrm{~b}^{\mathrm{a}}$ & $2.898(9)$ & $\mathrm{Cu} 1-\mathrm{N} 37$ & $2.025(3)$ \\
\hline $\mathrm{Cu} 1-\mathrm{N} 12$ & $1.942(3)$ & & \\
\hline \multicolumn{4}{|l|}{ Bond angles } \\
\hline $\mathrm{O} 51-\mathrm{Cu} 1-\mathrm{O} 61 \mathrm{a}^{\mathrm{a}}$ & $158.2(2)$ & $\mathrm{N} 32-\mathrm{Cu} 1-\mathrm{O} 61 \mathrm{a}^{\mathrm{a}}$ & $73.77(17)$ \\
\hline $\mathrm{O} 51-\mathrm{Cu} 1-\mathrm{O} 61 \mathrm{~b}^{\mathrm{a}}$ & 176.43(19) & $\mathrm{N} 32-\mathrm{Cu} 1-\mathrm{O} 61 \mathrm{~b}^{\mathrm{a}}$ & $90.6(2)$ \\
\hline $\mathrm{O} 51-\mathrm{Cu} 1-\mathrm{N} 12$ & $97.54(11)$ & $\mathrm{N} 37-\mathrm{Cu} 1-\mathrm{O} 61 \mathrm{a}^{\mathrm{a}}$ & $70.5(2)$ \\
\hline $\mathrm{O} 51-\mathrm{Cu} 1-\mathrm{N} 17$ & $83.98(11)$ & $\mathrm{N} 37-\mathrm{Cu} 1-\mathrm{O} 61 \mathrm{~b}^{\mathrm{a}}$ & $90.88(19)$ \\
\hline $\mathrm{O} 51-\mathrm{Cu} 1-\mathrm{N} 32$ & $91.14(11)$ & $\mathrm{N} 12-\mathrm{Cu} 1-\mathrm{N} 17$ & $80.97(12)$ \\
\hline $\mathrm{O} 51-\mathrm{Cu} 1-\mathrm{N} 37$ & $92.46(13)$ & $\mathrm{N} 12-\mathrm{Cu} 1-\mathrm{N} 32$ & $99.14(12)$ \\
\hline $\mathrm{N} 12-\mathrm{Cu} 1-\mathrm{O} 61 \mathrm{a}^{\mathrm{a}}$ & $100.3(2)$ & $\mathrm{N} 12-\mathrm{Cu} 1-\mathrm{N} 37$ & $169.78(14)$ \\
\hline $\mathrm{N} 12-\mathrm{Cu} 1-\mathrm{O} 61 \mathrm{~b}^{\mathrm{a}}$ & $79.08(18)$ & $\mathrm{N} 17-\mathrm{Cu} 1-\mathrm{N} 32$ & $175.09(12)$ \\
\hline $\mathrm{N} 17-\mathrm{Cu} 1-\mathrm{O} 61 \mathrm{a}^{\mathrm{a}}$ & $111.08(16)$ & $\mathrm{N} 17-\mathrm{Cu} 1-\mathrm{N} 37$ & $98.06(13)$ \\
\hline $\mathrm{N} 17-\mathrm{Cu} 1-\mathrm{O} 61 \mathrm{~b}^{\mathrm{a}}$ & $94.3(2)$ & $\mathrm{N} 32-\mathrm{Cu} 1-\mathrm{N} 37$ & $82.70(13)$ \\
\hline
\end{tabular}

${ }^{a}$ The weakly coordinated perchlorate ion was refined with a disorder model. O61a and O61b refer to two positions in this model with an occupancy of $0.59(1): 0.41(1)$.

more detailed description of the oxidative coupling equipment and the handling of the data has been given earlier [24,28].

\section{Results and discussion}

\subsection{Description of the structures}

\subsection{1. $\left[\mathrm{Cu}(\mathrm{pr} 2 \mathrm{pz})\left(\mathrm{ClO}_{4}\right)_{2}\right](\mathrm{A})$}

The molecular structure of $\left[\mathrm{Cu}(\mathrm{pr} 2 \mathrm{pz})\left(\mathrm{ClO}_{4}\right)_{2}\right]$ in the crystal [32] is depicted in Fig. 3. Crystallographic data are presented in Table 2, selected bond distances and bond angles are presented in Table 3. The copper(II) ion is in a distorted octahedral geometry, with the equatorial plane formed by four nitrogen donor atoms from the ligand: two pyrazole $\mathrm{N}$-atoms, $\mathrm{N} 12$ and $\mathrm{N} 32$, at distances of $1.942(3)$ and 1.971(3) $\AA$, respectively, and two secondary amine atoms, N17 and N37, at distances of 2.044(3) and 2.025(3) $\AA$, respectively. The deviation of the nitrogen atoms from the least-square $\mathrm{CuN}_{4}$ plane

Table 4

Selected distances $(\AA)$ and angles $\left({ }^{\circ}\right)$ for $\left[\mathrm{Cu}_{2}(\operatorname{pr} 4 \mathrm{pz} 22) \mathrm{Cl}_{4}\right](\mathrm{MeOH})(\mathbf{B})$

\begin{tabular}{lrlr}
\hline $\begin{array}{l}\text { Bond lengths } \\
\mathrm{Cu} 1-\mathrm{Cu} 1 \mathrm{a}\end{array}$ & $4.8132(4)$ & $\mathrm{Cu} 1-\mathrm{Cl} 2$ & $2.5388(6)$ \\
$\mathrm{Cu} 1-\mathrm{Cl} 3$ & $2.2431(5)$ & $\mathrm{Cu} 1-\mathrm{N} 12$ & $2.0803(17)$ \\
$\mathrm{Cu} 1-\mathrm{N} 22$ & $2.0849(17)$ & $\mathrm{Cu} 1-\mathrm{N} 27$ & $2.0688(18)$ \\
Bond angles & & & \\
$\mathrm{Cl} 2-\mathrm{Cu} 1-\mathrm{Cl} 3$ & $97.14(2)$ & $\mathrm{Cl} 2-\mathrm{Cu} 1-\mathrm{N} 12$ & $96.15(5)$ \\
$\mathrm{Cl} 2-\mathrm{Cu} 1-\mathrm{N} 22$ & $96.31(5)$ & $\mathrm{Cl} 2-\mathrm{Cu} 1-\mathrm{N} 27$ & $92.84(5)$ \\
$\mathrm{Cl} 3-\mathrm{Cu} 1-\mathrm{N} 12$ & $101.93(5)$ & $\mathrm{Cl} 3-\mathrm{Cu} 1-\mathrm{N} 22$ & $99.64(5)$ \\
$\mathrm{Cl} 3-\mathrm{Cu} 1-\mathrm{N} 27$ & $169.96(5)$ & $\mathrm{N} 12-\mathrm{Cu} 1-\mathrm{N} 22$ & $153.46(7)$ \\
$\mathrm{N} 12-\mathrm{Cu} 1-\mathrm{N} 27$ & $77.96(7)$ & $\mathrm{N} 22-\mathrm{Cu} 1-\mathrm{N} 27$ & $78.10(7)$ \\
\hline
\end{tabular}

Symmetry code: $a=1-x, 1-y, 1-z$.
Table 5

Selected distances $(\AA)$ and angles $\left(^{\circ}\right)$ for $\left[\mathrm{Cu}_{4}([18] \text { py } 2 \mathrm{pz})_{2}(\mu-\mathrm{Cl})_{4} \mathrm{Cl}_{2}\right]^{2+}$ (C)

\begin{tabular}{|c|c|c|c|}
\hline \multicolumn{4}{|l|}{ Bond lengths } \\
\hline $\mathrm{Cu} 1-\mathrm{Cula}$ & $3.8484(10)$ & $\mathrm{Cu} 1-\mathrm{Cu} 2$ & $4.8506(10)$ \\
\hline $\mathrm{Cu} 1-\mathrm{N} 12$ & $1.975(4)$ & $\mathrm{Cu} 2-\mathrm{N} 32$ & $2.024(5)$ \\
\hline $\mathrm{Cu} 1-\mathrm{N} 17$ & $2.089(4)$ & $\mathrm{Cu} 2-\mathrm{N} 37$ & $2.090(5)$ \\
\hline $\mathrm{Cu} 1-\mathrm{N} 21$ & $1.957(4)$ & $\mathrm{Cu} 2-\mathrm{N} 41$ & $2.001(5)$ \\
\hline $\mathrm{Cu} 1-\mathrm{Cl} 3$ & $2.8487(14)$ & $\mathrm{Cu} 2-\mathrm{Cl} 3$ & $2.3230(14)$ \\
\hline $\mathrm{Cu} 1-\mathrm{Cl} 4$ & $2.2655(14)$ & $\mathrm{Cu} 2-\mathrm{Cl} 5$ & $2.3761(16)$ \\
\hline $\mathrm{Cu} 1-\mathrm{Cl} 4 \mathrm{a}$ & $3.0297(14)$ & & \\
\hline \multicolumn{4}{|l|}{ Bond angles } \\
\hline $\mathrm{Cl} 3-\mathrm{Cu} 1-\mathrm{Cl} 4$ & $90.15(5)$ & $\mathrm{N} 21-\mathrm{Cu} 1-\mathrm{Cl} 4 \mathrm{a}$ & $84.38(13)$ \\
\hline $\mathrm{Cl} 3-\mathrm{Cu} 1-\mathrm{Cl} 4 \mathrm{a}$ & $171.71(4)$ & $\mathrm{Cl} 3-\mathrm{Cu} 2-\mathrm{Cl} 5$ & $113.14(6)$ \\
\hline $\mathrm{Cl} 3-\mathrm{Cu} 1-\mathrm{N} 12$ & $98.68(13)$ & $\mathrm{Cl} 3-\mathrm{Cu} 2-\mathrm{N} 32$ & $100.00(13)$ \\
\hline $\mathrm{Cl} 3-\mathrm{Cu} 1-\mathrm{N} 17$ & $98.71(13)$ & $\mathrm{Cl} 3-\mathrm{Cu} 2-\mathrm{N} 37$ & $136.58(14)$ \\
\hline $\mathrm{Cl} 3-\mathrm{Cu} 1-\mathrm{N} 21$ & $87.80(13)$ & $\mathrm{Cl} 3-\mathrm{Cu} 2-\mathrm{N} 41$ & $94.10(15)$ \\
\hline $\mathrm{Cl} 4-\mathrm{Cu} 1-\mathrm{N} 12$ & $98.90(14)$ & $\mathrm{Cl} 5-\mathrm{Cu} 2-\mathrm{N} 32$ & $93.68(14)$ \\
\hline $\mathrm{Cl} 4-\mathrm{Cu} 1-\mathrm{N} 17$ & $170.98(13)$ & $\mathrm{Cl} 5-\mathrm{Cu} 2-\mathrm{N} 37$ & $110.16(14)$ \\
\hline $\mathrm{Cl} 4-\mathrm{Cu} 1-\mathrm{N} 21$ & $95.71(13)$ & $\mathrm{Cl} 5-\mathrm{Cu} 2-\mathrm{N} 41$ & $92.42(14)$ \\
\hline $\mathrm{Cl} 4-\mathrm{Cu} 1-\mathrm{Cl} 4 \mathrm{a}$ & $87.92(5)$ & $\mathrm{N} 32-\mathrm{Cu} 2-\mathrm{N} 37$ & $80.00(19)$ \\
\hline $\mathrm{N} 12-\mathrm{Cu} 1-\mathrm{N} 17$ & $81.56(18)$ & $\mathrm{N} 32-\mathrm{Cu} 2-\mathrm{N} 41$ & $160.99(19)$ \\
\hline $\mathrm{N} 12-\mathrm{Cu} 1-\mathrm{N} 21$ & $163.98(19)$ & $\mathrm{N} 37-\mathrm{Cu} 2-\mathrm{N} 41$ & $80.99(19)$ \\
\hline $\mathrm{N} 12-\mathrm{Cu} 1-\mathrm{Cl} 4 \mathrm{a}$ & $89.59(13)$ & $\mathrm{Cu} 1-\mathrm{Cl} 3-\mathrm{Cu} 2$ & $139.19(6)$ \\
\hline $\mathrm{N} 17-\mathrm{Cu} 1-\mathrm{N} 21$ & $82.98(17)$ & $\mathrm{Cu} 1-\mathrm{Cl} 4-\mathrm{Cu} 1 \mathrm{a}$ & $92.08(5)$ \\
\hline $\mathrm{N} 17-\mathrm{Cu} 1-\mathrm{Cl} 4 \mathrm{a}$ & $83.07(13)$ & & \\
\hline
\end{tabular}

varies from $-0.122(3)$ to $+0.143(3) \AA$. Oxygen atoms from two mono-coordinated perchlorate anions occupy the axial positions. One axial position is occupied by the oxygen $\mathrm{O} 51$ atom at a distance of 2.451(3) $\AA$, the other perchlorate anion is disordered and the $\mathrm{Cu}-\mathrm{O}$ distance is longer $(2.924(8)$ and $2.898(9) \AA$ for the disorder

Table 6

Selected distances $(\AA)$ and angles $\left({ }^{\circ}\right)$ for $\left[\mathrm{Cu}_{2}\left([18]\right.\right.$ py2pz) $\left.\left(\mathrm{CH}_{3} \mathrm{CN}\right)_{4}\left(\mathrm{ClO}_{4}\right)_{2}\right]\left(\mathrm{ClO}_{4}\right)_{2} \cdot 1.6 \mathrm{CH}_{3} \mathrm{CN}(\mathbf{D})$

Bond lengths

$\mathrm{Cu} 1-\mathrm{Cu} 2$

$\mathrm{Cu} 1-\mathrm{N} 12$

$7.2352(7)$

$\mathrm{Cu} 2-\mathrm{N} 32$

$\mathrm{Cu} 2-\mathrm{N} 37$

$2.060(3)$

$\mathrm{Cu} 2-\mathrm{N} 41$

$1.986(4)$

$\mathrm{Cu} 1-\mathrm{N} 21$

$2.024(4)$

$\mathrm{Cu} 1-\mathrm{N} 70$

2.555(4)

$\mathrm{Cu} 2-\mathrm{N} 80$

$\mathrm{Cu} 2-\mathrm{N} 83$

Cu2-O61

2.000(3)

2.055(3)

1.976(3)

2.020(4)

2.311(4)

Cu1-O51

2.383(3)

Bond angles

O51-Cu1-N12

O51-Cu1-N17

O51-Cu1-N21

O51-Cu1-N70

O51-Cu1-N73

N12-Cu1-N17

N12-Cu1-N21

N12-Cu1-N70

N12-Cu1-N73

N17-Cu1-N21

N17-Cu1-N70

N17-Cu1-N73

N21-Cu1-N70

N21-Cu1-N73

N70-Cu1-N73

92.94(13)

102.11(13)

92.24(13)

$84.70(13)$

$168.47(12)$

81.11(14)

$164.29(14)$

100.91(14)

$89.28(13)$

83.30(14)

$172.84(14)$

$89.40(13)$

94.33(15)

$88.59(14)$

83.77(14)

\begin{tabular}{lr}
$\mathrm{O} 61-\mathrm{Cu} 2-\mathrm{N} 32$ & $83.14(12)$ \\
$\mathrm{O} 61-\mathrm{Cu} 2-\mathrm{N} 37$ & $82.84(12)$ \\
$\mathrm{O} 61-\mathrm{Cu} 2-\mathrm{N} 41$ & $85.74(12)$ \\
$\mathrm{O} 61-\mathrm{Cu} 2-\mathrm{N} 80$ & $82.53(13)$ \\
$\mathrm{O} 61-\mathrm{Cu} 2-\mathrm{N} 83$ & $170.47(12)$ \\
$\mathrm{N} 32-\mathrm{Cu} 2-\mathrm{N} 37$ & $82.30(13)$ \\
$\mathrm{N} 32-\mathrm{Cu} 2-\mathrm{N} 41$ & $162.31(14)$ \\
$\mathrm{N} 32-\mathrm{Cu} 2-\mathrm{N} 80$ & $98.86(14)$ \\
$\mathrm{N} 32-\mathrm{Cu} 2-\mathrm{N} 83$ & $104.64(14)$ \\
$\mathrm{N} 37-\mathrm{Cu} 2-\mathrm{N} 41$ & $82.70(14)$ \\
$\mathrm{N} 37-\mathrm{Cu} 2-\mathrm{N} 80$ & $165.07(14)$ \\
$\mathrm{N} 37-\mathrm{Cu} 2-\mathrm{N} 83$ & $103.44(14)$ \\
$\mathrm{N} 41-\mathrm{Cu} 2-\mathrm{N} 80$ & $93.26(14)$ \\
$\mathrm{N} 41-\mathrm{Cu} 2-\mathrm{N} 83$ & $87.94(14)$ \\
$\mathrm{N} 80-\mathrm{Cu} 2-1 \mathrm{~N} 83$ & $90.73(14)$ \\
\hline
\end{tabular}




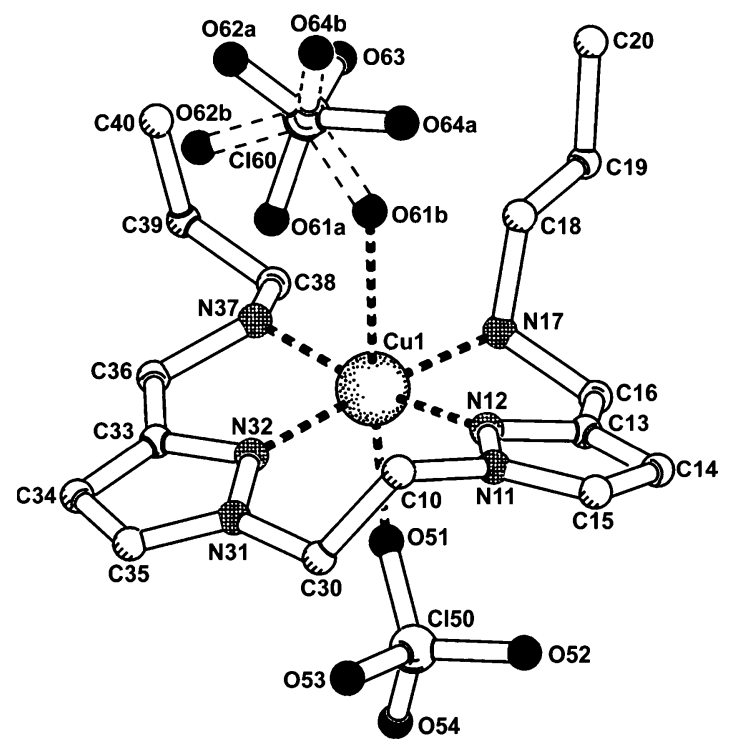

Fig. 3. PLUTON [32] plot of the molecular structure of $\left[\mathrm{Cu}(\mathrm{pr} 2 \mathrm{pz})\left(\mathrm{ClO}_{4}\right)_{2}\right](\mathbf{A})$. Hydrogen atoms are omitted for clarity.

positions $\mathrm{O} 61 \mathrm{a}$ and $\mathrm{O} 61 \mathrm{~b})$. The average in-plane $\mathrm{Cu}-\mathrm{N}$ distances are in the normal range for $\mathrm{CuN}_{4}$ squareplanar chromophores. The elongation of the $\mathrm{Cu}-\mathrm{O}$ distances for the axially coordinated perchlorate anions is common for $\mathrm{Cu}(\mathrm{II})$. The $\mathrm{Cu} 1-\mathrm{O} 51$ distance of 2.451(3) $\AA$ lies in the normal range for $\mathrm{Cu}-\mathrm{O}$ distances along the axial Jahn-Teller axis [33]. The other axial position is occupied by the perchlorate $\mathrm{O} 61$ atom at a rather large distance of approximately $2.9 \AA$ (in comparison with the $\mathrm{Cu} 1-\mathrm{O} 51$ distance of 2.451(3) $\AA$ ). In this case the perchlorate anion can be best regarded as semi-coordinated to the copper ion. A deviation from the in-plane right angles is observed for the $\mathrm{CuN}_{4}$ equatorial plane, caused by the ligand bites. The angles $\mathrm{N} 12-\mathrm{Cu} 1-\mathrm{N} 17$ and $\mathrm{N} 32-\mathrm{Cu} 1-\mathrm{N} 37$ of $80.97(12)^{\circ}$ and $82.70(13)^{\circ}$, respectively are smaller than $90^{\circ}$, which is caused by the strain due to the three-bond ligand bites. This in turn leads to an increase of the coordination angles N12-Cu1-N32 and N17-Cu1-N37, (99.14(12) and $\left.98.06(13)^{\circ}\right)$. The ligand forms an uncommon sevenatom chelate ring with the copper ion via N17 and N37. The sum of the plane angles is $360.87^{\circ}$, only slightly more than $360^{\circ}$.

\subsection{2. $\left[\mathrm{Cu}_{2}([22] p r 4 p z) \mathrm{Cl}_{4}\right](\mathrm{MeOH})(\boldsymbol{B})$}

A molecular plot of $\left[\mathrm{Cu}_{2}([22] \mathrm{pr} 4 \mathrm{pz}) \mathrm{Cl}_{4}\right]$ is given in Fig. 4. Crystallographic data are presented in Table 2. Selected bond distances and bond angles are presented in Table 4. The asymmetric unit comprises half a molecule $\left[\mathrm{Cu}_{2}([22] \mathrm{pr} 4 \mathrm{pz}) \mathrm{Cl}_{4}\right](\mathrm{MeOH})$, the other half of the molecule is generated through a center of symmetry. The $\mathrm{Cu}$ (II) ion is in an $\mathrm{N}_{3} \mathrm{Cl}_{2}$ environment, which can be best described as distorted square pyramidal with a $\tau$ value of 0.27 , indicating a geometry closer to a regular square pyramid $(\tau=0)$ than to a regular trigonal

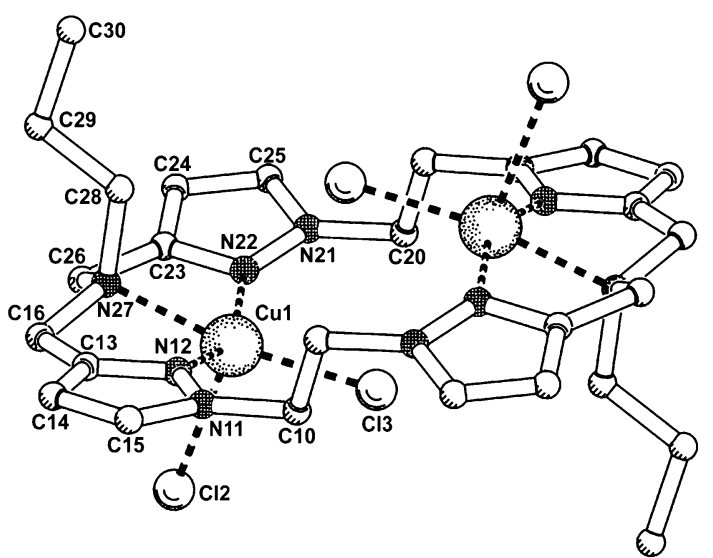

Fig. 4. PLUTON [32] plot of the molecular structure of $\left[\mathrm{Cu}_{2}([22] \mathrm{pr} 4 \mathrm{pz}) \mathrm{Cl}_{4}\right](\mathbf{B})$. Hydrogen atoms are omitted for clarity.

bipyramid $(\tau=1)$ [34]. The equatorial positions are occupied by the tertiary amine $\mathrm{N} 27$ atom at a distance of 2.0688(18) $\AA$, the two pyrazole atoms, N12 and N22 at distances of respectively 2.0803(17) and 2.0849(17) $\AA$, and the $\mathrm{Cl} 3$ atom at a distance of 2.2431(5) $\AA$. These inplane $\mathrm{Cu}-\mathrm{N}$ distances are in the normal range for $\mathrm{Cu}-$ $\mathrm{N}$ bonds in the plane of square-planar chromophores. The axial position is occupied by the other chloride atom $\mathrm{Cl} 2$ at a distance of 2.5388(6) $\AA$. The Jahn-Teller distortion, often seen in $\mathrm{Cu}(\mathrm{II})$ complexes [33], causes the substantial elongation of the $\mathrm{Cu} 1-\mathrm{Cl} 2$ bond compared to the in-plane $\mathrm{Cu} 1-\mathrm{Cl} 3$ bond. The small N27$\mathrm{Cu} 1-\mathrm{N} 12$ and $\mathrm{N} 27-\mathrm{Cu} 1-\mathrm{N} 22$ angles of respectively $77.96(7)^{\circ}$ and $78.10(7)^{\circ}$ are imposed by the three-bond ligand bites. The macrocycle adopts a sort of anti(chair)-conformation, which puts the $\mathrm{Cu}$ (II) ions ( $\mathrm{Cu} 1$ and $\mathrm{Cula}$ ) at different sides of the ring at a distance of 4.8132(4) $\AA$ with respect to each other. Non-coordinated (disordered) $\mathrm{MeOH}$ molecules are present in the crystal lattice, hydrogen bonded to the $\mathrm{Cl} 2$ atom.

\subsection{3. $\left[\mathrm{Cu}_{4}([18] \mathrm{py} 2 \mathrm{pz})_{2}(\mu-\mathrm{Cl})_{4} \mathrm{Cl}_{2}\right] \mathrm{Cl}_{2}(\mathrm{C})$}

The molecular plot of the cation $\left[\mathrm{Cu}_{4}([18] \mathrm{py} 2 \mathrm{pz})_{2}(\mu-\right.$ $\left.\mathrm{Cl})_{4} \mathrm{Cl}_{2}\right]^{2+}$ is shown in Fig. 5. Crystallographic data are presented in Table 2. Selected bond lengths and bond angles are presented in Table 5. The cation of complex $\mathbf{C}$ contains four copper centers. Two bridging $\mathrm{Cl}$ atoms connect the two central copper atoms to form a centrosymmetric four membered ring. The bridging $\mathrm{Cl} 4-\mathrm{Cu}$ distances of 2.2655(14) and 3.0297(14) $\AA$ indicate a very asymmetric bridge with one $\mathrm{Cl}$ atom only weakly bound. The distorted octahedral coordination environment of these central copper atoms $\mathrm{Cu} 1$ is completed with a third chloride $\mathrm{Cl} 3$ atom $(\mathrm{Cu} 1-\mathrm{Cl} 3$ $2.8487(14) \AA$ ) and three nitrogen atoms from the [18]py2pz ligand, N21 from a pyridine residue $(\mathrm{Cu} 1-$ N21 1.957(4) A), N12 from a pyrazole residue (Cu1$\mathrm{N} 121.975(4) \AA$ ) and the tertiary amine nitrogen N17 (Cu1-N17 2.089(4) $\AA$ ). Thus, the central plane of the 


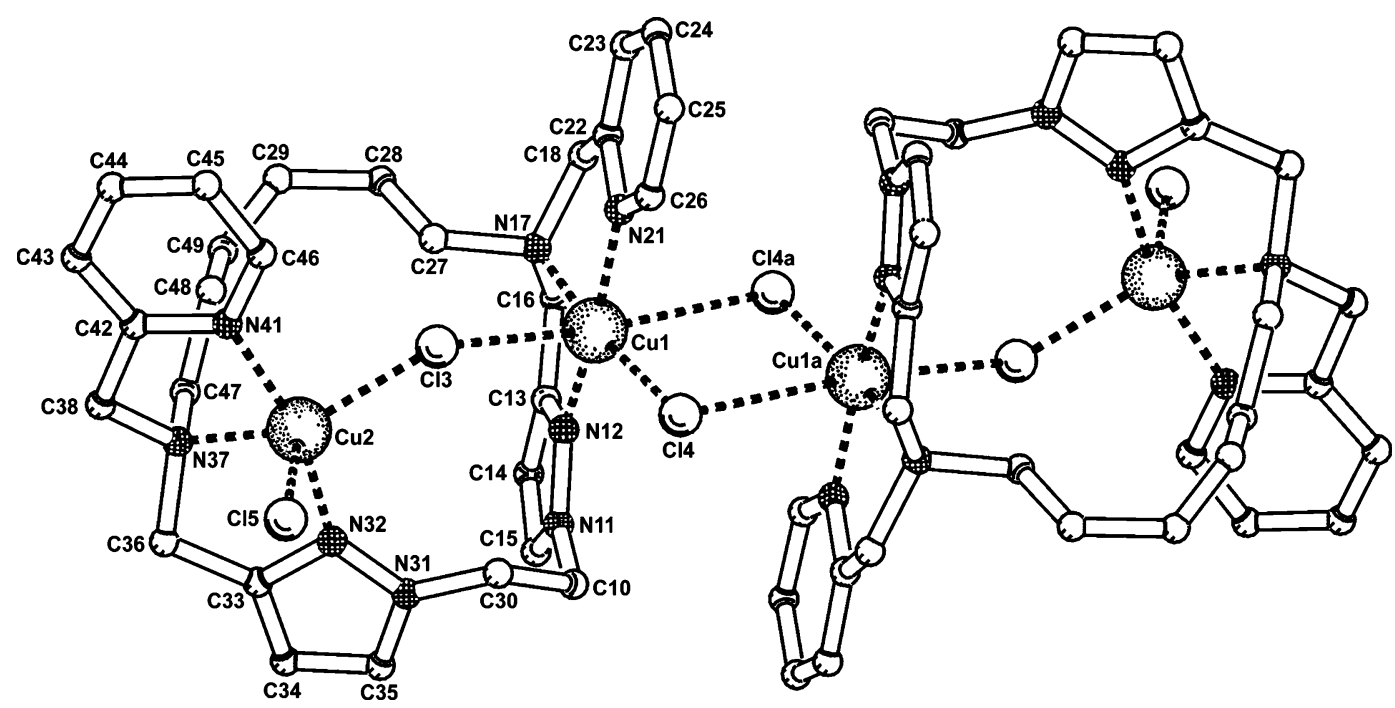

Fig. 5. PLUTON [32] plot of the molecular structure of the complex cation $\left[\mathrm{Cu}_{4}([18] \mathrm{py} 2 \mathrm{pz})_{2}(\mu-\mathrm{Cl})_{4} \mathrm{Cl}_{2}\right]^{2+}(\mathbf{C})$. Hydrogen atoms are omitted for clarity.

octahedron is formed by $\mathrm{Cl} 4$ and the three nitrogen atoms. Values of $81.56(18)$ and $82.98(17)^{\circ}$ for the chelating $\mathrm{N}-\mathrm{Cu}-\mathrm{N}$ cis angles and 95.71(13) and $98.90(14)^{\circ}$ for the non-chelating $\mathrm{N}-\mathrm{Cu} 1-\mathrm{Cl} 4$ cis angles resulted in an angle sum of $359.15^{\circ}$. The weakly bound chlorides $\mathrm{Cl} 3$ and $\mathrm{Cl}^{\prime}$ are located in axial positions of the octahedron with an angle $\mathrm{Cl} 3-\mathrm{Cul}-\mathrm{Cl} 4 \mathrm{a}$ of 171.71(4) $)^{\circ}$. Chloride $\mathrm{Cl} 3$ is bridging $\mathrm{Cu} 1$ with the crystallographically independent $\mathrm{Cu} 2$. With distances $\mathrm{Cl} 3-\mathrm{Cu} 1$ of $2.8487(14)$ and $\mathrm{Cl} 3-\mathrm{Cu} 2$ of $2.3230(14) \AA$, this bridge is asymmetric as the $\mathrm{Cu} 1-\mathrm{Cl} 4-\mathrm{Cula}$ bridge, but the asymmetry is less extreme. $\mathrm{Cu} 2$ has a fivefold coordination environment with a second chlorine $\mathrm{Cl} 5$ $(\mathrm{Cu} 2-\mathrm{Cl} 52.3761(16) \AA)$ and three nitrogens of the [18]py2pz ligand, N41 from a pyridine residue $(\mathrm{Cu} 2-$ N41 2.001(5) $\AA$ ), N32 from a pyrazole residue $(\mathrm{Cu} 2-$ N32 2.024(5) $\AA$ ), and the tertiary amine nitrogen N37 (Cu2-N37 2.090(5) A). The distances are thus in the same range as for the six coordinated $\mathrm{Cu} 1$. The geometry of $\mathrm{Cu} 2$ is best described as intermediate between square pyramidal and trigonal bipyramidal, resulting in a $\tau$ value [34] of 0.41 . The metal-metal distance between the symmetry related $\mathrm{Cu} 1$ and $\mathrm{Cu} 1 \mathrm{a}$ is 3.8484(10) $\AA$, and thereby much shorter than the distance of $4.8506(10) \AA$ between $\mathrm{Cu} 1$ and $\mathrm{Cu} 2$. While $\mathrm{Cu} 1$ and Cula are bridged by two chlorine atoms, the bridge between $\mathrm{Cu} 1$ and $\mathrm{Cu} 2$ consists of one chlorine and the [18]py2pz ligand.

\subsection{4. $\left[\mathrm{Cu}_{2}([\mathrm{l}] \mathrm{py} 2 \mathrm{pz})\left(\mathrm{CH}_{3} \mathrm{CN}\right)_{4}\left(\mathrm{ClO}_{4}\right)_{2}\right]\left(\mathrm{ClO}_{4}\right)_{2-}$ $\left(\mathrm{CH}_{3} \mathrm{CN}\right)_{1.6}(\boldsymbol{D})$}

A projection of the molecular structure of $\left[\mathrm{Cu}_{2}([18] \text { py2pz })\left(\mathrm{CH}_{3} \mathrm{CN}\right)_{4}\left(\mathrm{ClO}_{4}\right)_{2}\right]^{2+}$ is depicted in Fig. 6. Crystallographic data are presented in Table 2, selected bond distances and bond angles are presented in Table 6. Both copper(II) ions in the complex have a distorted octahedral geometry, with the equatorial plane formed by three nitrogen donor atoms from the macrocyclic ligand and one acetonitrile molecule. A second acetonitrile molecule and a mono-coordinated perchlorate anion occupy the axial positions. The approximate twofold symmetry of the dimeric complex is broken by the axial ligands, which have interchanged their positions. The equatorial plane of the $\mathrm{Cul}$ ion is formed by the pyridine N21 atom at a distance of $1.986(4) \AA$, the pyrazole N12 atom at a distance of 2.005(4) $\AA$, the tertiary amine $\mathrm{N} 17$ atom at a distance of 2.060(3) $\AA$ and the acetonitrile N70 atom at a distance of 2.024(4) $\AA$. The corresponding distances at $\mathrm{Cu} 2$ are 1.976(3), 2.000(3), 2.055(3) and 2.020(4) ^, respectively. The axial positions are occupied by the second acetonitrile N73 atom at a distance of 2.555(4) $\AA$ and the perchlorate O51 atom at a distance of 2.383(3) $\AA$. At $\mathrm{Cu} 2$ the distances are correspondingly 2.311(4) and 2.718(3) A. The in-plane $\mathrm{Cu}-\mathrm{N}$ distances for $\mathrm{Cu} 1$ and $\mathrm{Cu} 2$ are in the normal range for $\mathrm{CuN}_{4}$ square-planar chromophores. The elongation of the $\mathrm{Cu}-\mathrm{N}$ distance for the axially coordinated acetonitrile molecule in comparison with the acetonitrile molecule in the equatorial position is common for $\mathrm{Cu}(\mathrm{II})$. The perchlorate distances to $\mathrm{Cu} 1$ and $\mathrm{Cu} 2$ are quite different. While the $\mathrm{Cu} 1-\mathrm{O} 51$ distance of 2.383(3) $\AA$ lies in the normal range for $\mathrm{Cu}-\mathrm{O}$ distances along the axial Jahn-Teller axis [33], the $\mathrm{Cu} 2-061$ distance of 2.718(3) $\AA$ is rather large. We therefore consider this perchlorate ion only semi-coordinated to $\mathrm{Cu} 2$. A deviation from the in-plane right angles is observed for the $\mathrm{CuN}_{4}$ equatorial planes. The chelated angles N12-Cu1-N17, N17-Cu1-N21, N32$\mathrm{Cu} 2-\mathrm{N} 37$, and $\mathrm{N} 37-\mathrm{Cu} 2-\mathrm{N} 41$ of $81.11(14), 83.30(14)$, $82.30(13)$ and $82.70(14)^{\circ}$ are significantly smaller than 


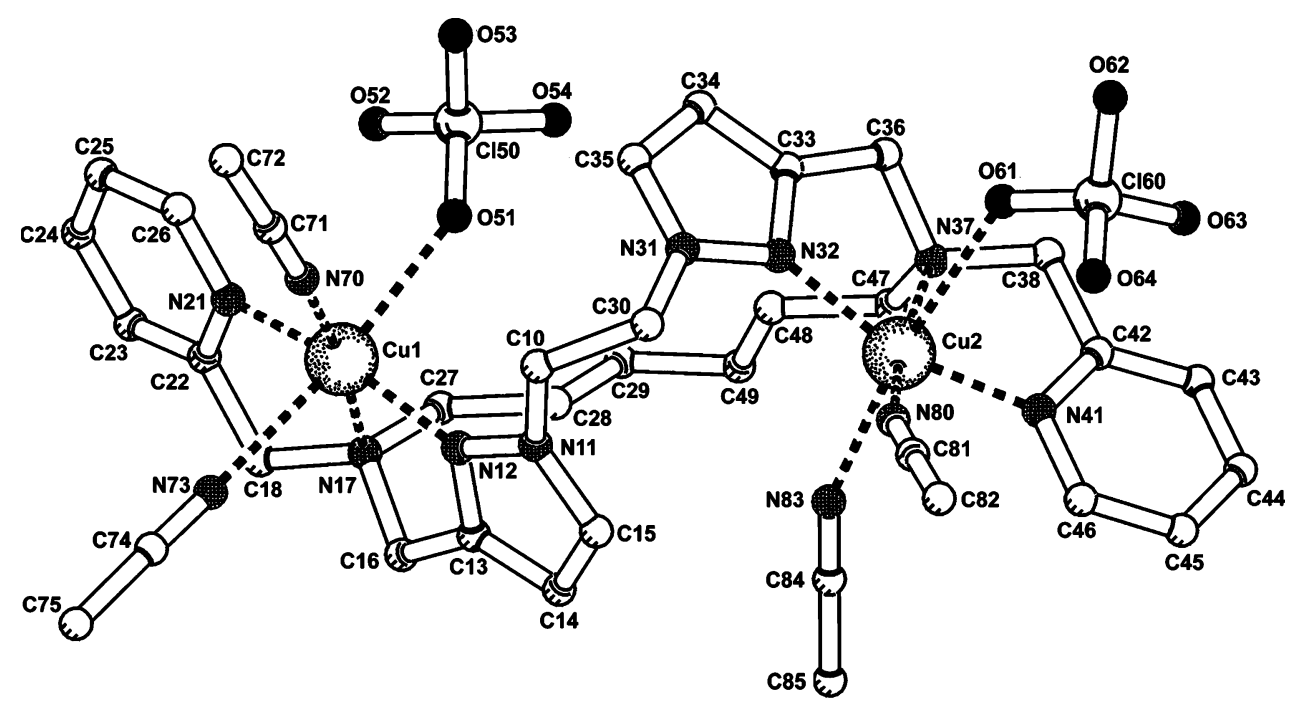

Fig. 6. PLUTON [32] plot of the molecular structure of the complex cation $\left[\mathrm{Cu}_{2}([18] \mathrm{py} 2 \mathrm{pz})\left(\mathrm{CH}_{3} \mathrm{CN}\right)_{4}\left(\mathrm{ClO}_{4}\right)_{2}\right]^{2+}(\mathbf{D})$. Hydrogen atoms are omitted for clarity.

$90^{\circ}$. This in turn leads to the larger coordination angles of the opposite cis angles. The sum of the plane angles is $365.66^{\circ}$ for $\mathrm{Cu} 1$ and $357.14^{\circ}$ for $\mathrm{Cu} 2$. The lack of a bridging ligand [35] allows the macrocyclic ligand [18]py2pz to adopt an anti-conformation [36] holding the coppers at opposite sides of the macrocycle at a rather large distance of 7.2352(7) $\AA$. Further, two noncoordinating disordered perchlorate counter ions and 1.6 non-coordinated acetonitrile molecules are present in the crystal lattice per formula unit.

\subsubsection{Comparison of the structures}

The ligand pr2pz in compound $\mathbf{A}$ is the precursor of the macrocycle [22]pr4pz in compound $\mathbf{B}$. The precursor is still very flexible and forms a mononuclear complex with copper(II). The [22]pr4pz macrocycle is quite rigid. The copper ions are held at a distance of 4.8132(4) $\AA$ of each other. As is clear from compounds $\mathbf{C}$ and $\mathbf{D}$, the [18]py2pz macrocycle is much more flexible. In the synconformation the ligand holds the two copper ions at a distance of 4.8506(10) $\AA$ of each other, which is significantly shorter than the $\mathrm{Cu}-\mathrm{Cu}$ distance in the $\left[\mathrm{Cu}_{2}\left([18] \text { py2pz) }\left(\mathrm{CH}_{3} \mathrm{CN}\right)_{4}\left(\mathrm{ClO}_{4}\right)_{2}\right]^{2+}\right.$ cation of $7.2352(7) \AA$. This shortening is apparently caused by the presence of a bridging chloride atom between the two copper ions, as well as the syn-conformation of the macrocyclic ligand instead of the anti-conformation observed for complex D. No hydrogen bonding, or stacking interaction between the aromatic rings is observed in either $\mathbf{A}, \mathbf{C}$ or $\mathbf{D}$. Thus the crystal packing in the compounds is only due to van der Waals interactions.

\subsection{Spectroscopic data}

Relevant spectroscopic and analytical data of the coordination compounds $\mathbf{A}, \mathbf{B}, \mathbf{C}$ and $\mathbf{D}$ are listed in Table 1. All complexes give normal infrared spectra with vibrations characteristic for the ligands and counter ions present. The ligand field spectra, measured as solids with the diffuse-reflectance technique, give broad bands between 11.6 and $17.4 \times 10^{3} \mathrm{~cm}^{-1}$. The X-band EPR spectrum of $\mathbf{D}$ as a powder, recorded at room temperature and at $77 \mathrm{~K}$, is isotropic with a $g$ value of 2.1. The $\mathrm{X}$-band EPR spectra of $\mathbf{A}, \mathbf{B}$ and $\mathbf{C}$ recorded as powders, at room temperature and at $77 \mathrm{~K}$, show axial signals with $g_{\perp}$ values of $2.06,2.05$ and 2.03 and $g_{\|}$ values of 2.20, 2.17 and 2.22, respectively. Compound $\mathbf{A}$ shows a hyperfine splitting $A_{\|}$of 19.4 (mT). No hyperfine splittings of $\mathbf{B}$ and $\mathbf{C}$ are resolved and no triplet signals are detected. These data suggest an exchange interaction leading to exchange-narrowing. The EPR and ligand field spectra of the ligand pr2pz in solution treated with different equivalents of copper(II) perchlorate indicate that also in solution a mononuclear complex is present, even when two or more equivalents of copper salt are present. The intensity of the LF band at $16.5 \times 10^{3} \mathrm{~cm}^{-1}$ grows until 1 equiv. of copper salt is added to the ligand. Adding more equivalents of copper salt does neither result in an increase of intensity of the band at $16.5 \times 10^{3} \mathrm{~cm}^{-1}$, nor in the appearance of another band other than the absorption due to free copper salt. The X-band EPR spectra of pr2pz plus 1 equiv. of copper(II) perchlorate, taken as a frozen methanol solution at $77 \mathrm{~K}$ is similar to the EPR spectrum of the solid spectrum of $\mathbf{A}$, apart 
Table 7

Relevant spectroscopic data of: ligand $+2 \mathrm{Cu}\left(\mathrm{NO}_{3}\right)_{2}\left(2.5 \mathrm{mM}\right.$ in $\left.\mathrm{CH}_{3} \mathrm{CN}\right)$

\begin{tabular}{llll}
\hline Ligand & Color of the solution & Ligand field $\left(10^{3} \mathrm{~cm}^{-1}\right)$ in $\mathrm{CH} 3 \mathrm{CN}$ & $\mathrm{EPR}$ in $\mathrm{CH}_{3} \mathrm{CN}, 77 \mathrm{~K}$ \\
\hline$[22] \mathrm{py} 4 \mathrm{pz}$ & greenish & 10.9 & $g_{\perp}=2.13$ \\
{$[22] \mathrm{pr} 4 \mathrm{pz}$} & dark blue & 14.5 & $g_{\perp}=2.12$ \\
{$[20] \mathrm{py} 2 \mathrm{pz}$} & dark blue & 14.8 & $g_{\perp}=2.11$ \\
{$[18] \mathrm{py} 2 \mathrm{pz}$} & dark blue & 14.8 & $g_{\perp}=2.12$ \\
Py2pz & dark blue & 15.1 & $g_{\perp}=2.11$ \\
Pr2pz & dark blue & 14.8 & $g_{\perp}=2.12$ \\
\hline
\end{tabular}

from some super hyperfine splitting superimposed on the axial signal. When more than 1 equiv. of copper salt is added to pr2pz signals of a copper(II) perchlorate methanol complex appear.

\subsection{Oxidative coupling reactions}

\subsubsection{Spectroscopic data}

For the oxidative coupling experiments acetonitrile solutions of the ligands with 2 equiv. of copper(II) nitrate were used. EPR and ligand field data of these solutions are listed in Table 7. All complexes have a single broad band in their ligand field spectrum around $14.8 \times 10^{3} \mathrm{~cm}^{-1}$, except the complex of [22]py2pz which has a broad band at $10.9 \times 10^{3} \mathrm{~cm}^{-1}$. All frozensolution EPR spectra of the complex solutions look remarkably similar with isotropic signals with $g$ values of 2.1. For the precursor ligands pr2pz and py2pz also titrations of the ligands in acetonitrile with different equivalents of copper(II) nitrate were performed. From the ligand field data it becomes clear that py2pz forms a dinuclear complex in solution, while pr2pz exists (mainly) as a mononuclear complex in solution.

\subsubsection{General observations}

The copper nitrate complexes with four macrocyclic ligands and two of their precursor ligands have been found to be active in the oxidative coupling reaction, but the relative activities of the complexes differ significantly (see Fig. 7). These dissimilarities can best be explained by the differences between the ligands and therefore the copper complexes. The polymerization of DMP is thought to involve a dinuclear copper intermediate [24]. A copper complex with two copper atoms preorganized in a relatively close proximity might facilitate the formation of such an intermediate. In the complexes of the macrocyclic ligands the copper atoms are already held closer together than in those of the precursors, but the flexibility of the macrocycles differs somewhat from each other and rather strongly with the precursors. The complexes differ also in the number of ligand nitrogen donors surrounding each copper atom. The coordination sphere around the copper in solution of the macrocyclic copper nitrate complexes is most likely completed with counter ions or acetonitrile molecules, which are replaced more easily by substrate or dioxygen molecules than the ligand nitrogen atoms. Although the precursor ligand $\mathrm{pr} 2 \mathrm{pz}$ forms a mononuclear complex in solution when no substrate or other bridging compound is available (see above), in the oxidative coupling experiments 2 equiv. of copper to $\mathrm{pr} 2 \mathrm{pz}$ have been added with the aim to induce possible dinucleation with DMP and dioxygen as bridges.

\subsubsection{Comparison of the activities}

The complexes with the macrocycles [18]py2pz and [20]py2pz have been found to be the most active (see Fig. 7). The ligands [18]py2pz and [20]py2pz differ from [22]py4pz in that one 'bispyrazole site' of the latter is replaced by an alkyl chain (hexyl in the case of [18]py2pz and octyl in the case of [20]py2pz). The difference in length of the alkyl chain does not seem to influence the catalytic activity. Whereas going from a $2 \times 4$ nitrogen donor ([22]py4pz) to a $2 \times 3$ nitrogen donor ([18]py $2 \mathrm{pz}$ and [20]py2pz) appears to have a dramatic influence on the catalytic activity, with [22]py4pz having a significantly lower activity. Apparently, the copper complexes are more active when more positions are available for the coordination of the substrate DMP. Additionally, in the ' $2 \times 3$ ' cases the copper ions are less sterically hindered by the ligand. Both the copper complexes of [18]py2pz and [20]py2pz were found to give the same activities. These copper complexes are rather flexible as can be seen from the structures of the complexes $\mathbf{C}$ and $\mathrm{D}$ where a bridging ligand reduces the $\mathrm{Cu}-\mathrm{Cu}$ distance from approximately 7 to $5 \AA$. The relatively small change in the alkyl chain length does not influence the

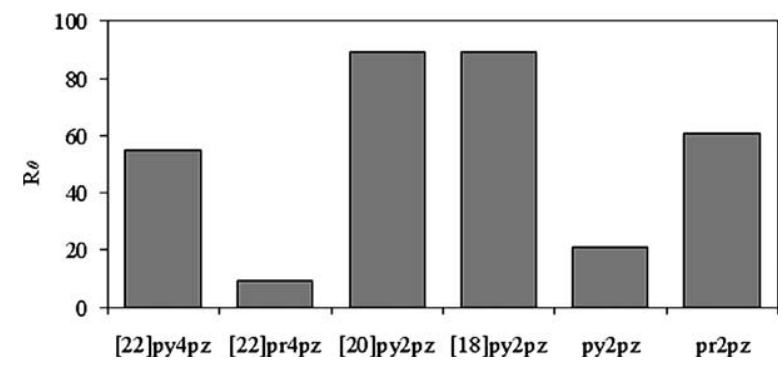

Fig. 7. Initial reaction rates, $R_{0}\left(10^{-5} \mathrm{~mol}^{-1} \mathrm{~s}^{-1}\right)$, of the DMP conversion catalyzed by $\mathrm{Cu}_{2}$ (ligand) $\left(\mathrm{NO}_{3}\right)_{4}$. 
activity significantly, probably due to a similar flexibility. The copper complex of [22]py4pz also possesses some flexibility as can be inferred from previously reported mepy22pz structures with variations in $\mathrm{Cu}-$ $\mathrm{Cu}$ distances from approximately 8.6 to $6.8 \AA$ [19]. These larger distances than in the [18]py2pz and [20]py $2 \mathrm{pz}$ complexes may also contribute to a lower activity, as it hampers the formation of a catalytically active dicopper species.

The low catalytic activity of the dicopper complex of [22]pr4pz compared to [22]py4pz is quite remarkable. The difference between [22]pr4pz and [22]py4pz being propyl groups instead of the exocyclic pyridines of the latter. The origin for the low activity of the complex with this $2 \times 3$ nitrogen donor ligand might be the coordination sphere around the copper induced by the ligand, lacking the adjustable pendant pyridine arm. From the crystal structure of $\mathbf{B}$ it can be seen that the three ligand nitrogen atoms lie approximately in one plane with the copper, holding the two coppers with the surrounding macrocycle firmly at a distance of 4.8132(4) $\AA$ of each other. This rigidity may hamper the oxidative coupling reaction, by preventing the complex to adopt the right conformation for the formation of a catalytically active dicopper species.

For the precursor ligands py $2 \mathrm{pz}$ and pr2pz a relatively low catalytic activity was expected because these ligands are less capable to hold the copper atoms already in close proximity compared to the complexes with the corresponding macrocyclic ligands. The copper complex of py $2 \mathrm{pz}$ (the precursor of [22]py4pz, [18]py2pz, and [20]py2pz) does show this expected low activity. In contrast the complex of pr2pz (the precursor of [22]pr4pz) has a relatively high activity. The increase in activity going from py $2 \mathrm{pz}$ to $\mathrm{pr} 2 \mathrm{pz}$ may result from the number of ligand nitrogen donors available for the copper atoms, being $2 \times 3$ for py $2 \mathrm{pz}$ and $2 \times 2$ for pr $2 \mathrm{pz}$. Of course it is not clear whether a dinuclear intermediate with pr2pz or py $2 \mathrm{pz}$ as dinucleating ligands is formed or a dinuclear intermediate existing of two mononuclear copper-ligand units coupled via the substrate and/or dioxygen. In comparing the activities of the complexes of the precursor ligands with those of the corresponding macrocyclic complexes, another factor to be taken into account is the influence of the type of amine nitrogens in the ligands, being tertiary for the macrocycles and secondary for the precursor ligands [37].

\subsection{4. $D M P$ and $D P Q$ ratios}

The percentages of DMP conversion and amounts of DPQ formed in the reactions are depicted in Fig. 8. The amount of DPQ formed is given in percentages of the initial amount of DMP. In most cases, the amount of DPQ formed lies in the same range for all the tested complexes, but is higher than obtained with the monodentate ligand $N$-methylimidazole [24,38]. This high

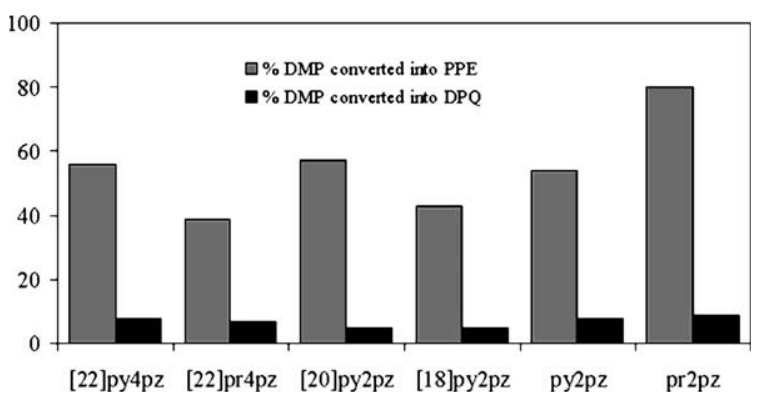

Fig. 8. Percentage of the converted amount of DMP and the amount of DPQ formed during the polymerization reaction catalyzed by $\mathrm{Cu}_{2}$ (ligand) $\left(\mathrm{NO}_{3}\right)_{4}$ complexes.

amount of DPQ is in contrast with the most recently proposed mechanism, where it is assumed that DPQforming species are mononuclear copper compounds. However, despite the use of ligands which were designed to form dinuclear copper complexes, it is still possible that in solution the copper ions are on such a large distance that part of the complex behaves as a mononuclear (DPQ-forming) species.

Although the initial reaction rates of the polymerization differ significantly, the total amount of converted DMP is almost the same for all reactions. It is interesting to note that when approximately $55-60 \%$ of the DMP is converted the reaction abruptly stops. The reason for this sudden stop might be the conversion of the catalyst into an inactive copper hydroxide complex, due to the water formed during the reaction. This decomposition of the active copper complex has also been observed with the analogous ligand etpy $24 \mathrm{pz}$ in another solvent system [39]. When an excess of $N$ methylimidazole, which is known to make the catalyst more stable in water [40], was added to a stopped reaction with [18]pr2pz as the ligand, new activity was observed and about $100 \%$ conversion of DMP could be reached. Furthermore, with only $\mathrm{N}$-methylimidazole as the ligand in the same solvent system an excess of $\mathrm{N}$ methylimidazole is required to obtain $100 \%$ DMP conversion [40], whereas in the present study only stoichiometric amounts of ligands were used.

\section{Concluding remarks}

Four new macrocyclic ligands, [22]py4pz, [22]pr4pz, [18]py2pz and [20]py2pz and two precursor ligands pr2pz and py2pz have been synthesized and four interesting single crystal X-ray structures have been obtained of the copper compounds with the ligands [22]pr4pz, [18]py2pz and pr2pz. The copper nitrate compounds of the new ligands appeared to be catalysts of variable activity for the oxidative coupling of DMP to PPE. With less nitrogen atoms available to coordinate the copper ions, somewhat higher activities were 
obtained. For all copper complexes a relatively high amount of DPQ was formed. Full conversion of the substrate DMP was not achieved, as in all cases the oxidative coupling abruptly stops before $60 \%$ conversion, probably due to the formation of inactive copper hydroxide species.

\section{Supplementary material}

Crystallographic data (excluding structure factors) have been deposited with the Cambridge Crystallographic Data Centre as supplementary publication no. CCDC-213518 compound A, -213519 compound B, -213520 compound C, -213521 compound D.

Copies of the data can be obtained free of charge on application to CCDC, 12 Union Road, Cambridge CB2 1EZ, UK (Fax: +44-1223/336-033; E-mail: deposit@ ccdc.cam.ac.uk or www: http://www.ccdc.cam.ac.uk.

\section{Acknowledgements}

This research has been financially supported by the Council for Chemical Sciences of the Netherlands Organization for Scientific Research (CW-NWO).

\section{References}

[1] K.A. Magnus, H. Ton-That, J.E. Carpenter, Chem. Rev. 94 (1994) 727.

[2] E.I. Solomon, U.M. Sundaram, T.E. Machonkin, Chem. Rev. 96 (1996) 2563.

[3] T. Klabunde, C. Eicken, J.C. Sacchettini, B. Krebs, Nat. Struct. Biol. 5 (1998) 1084.

[4] K.D. Karlin, Z. Tyeklar, Bioinorganic Chemistry of Copper, Chapman \& Hall, New York, 1993

[5] C. Eicken, B. Krebs, J.C. Sacchettini, Curr. Opin. Struct. Biol. 9 (1999) 677.

[6] A. Rompel, H. Fischer, D. Meiwes, K. Buldt Karentzopoulos, R. Dillinger, F. Tuczek, H. Witzel, B. Krebs, J. Biol. Inorg. Chem. 4 (1999) 56.

[7] S. Schindler, Eur. J. Inorg. Chem. (2000) 2311.

[8] K.D. Karlin, A.D. Zuberbühler, in: J. Reedijk, E. Bouwman (Eds.), Bioinorganic Catalysis, second ed., Marcel Dekker, New York, 1999, p. 469.

[9] L. Que, in: J. Reedijk, E. Bouwman (Eds.), Bioinorganic Catalysis, second ed., Marcel Dekker, New York, 1999, p. 269.

[10] N. Kitajima, Y. Moro-oka, Chem. Rev. 94 (1994) 737.

[11] N. Kitajima, Adv. Inorg. Chem. 39 (1994) 1.
[12] K.D. Karlin, S. Kaderli, A.D. Zuberbühler, Acc. Chem. Res. 30 (1997) 139.

[13] W.B. Tolman, Acc. Chem. Res. 30 (1997) 227.

[14] R.A. Sheldon, J.K. Kochi, Metal-Catalyzed Oxidations of Organic Compounds, Academic Press, New York, 1981.

[15] R.A. Sheldon, Bioinorganic Catalysis, Marcel Dekker, New York, 1993.

[16] E. Tsuchida, K. Yamamoto, Bioinorganic Catalysis, Marcel Dekker, New York, 1993.

[17] K.A. Magnus, H. Ton-That, J.E. Carpenter, in: K.D. Karlin, Z. Tyeklar (Eds.), Bioinorgainic Chemistry of Copper, Chapman \& Hall, New York, 1993, p. 143.

[18] J.E. Bol, W.L. Driessen, R.Y.N. Ho, B. Maase, L. Que, J. Reedijk, Angew. Chem., Int. Ed. Engl. 36 (1997) 998.

[19] R.H. Bode, J.E. Bol, W.L. Driessen, F.B. Hulsbergen, J. Reedijk, A.L. Spek, Inorg. Chem. 38 (1999) 1239.

[20] A.S. Hay, J. Polym. Sci. A 36 (1998) 505.

[21] H. Uyama, S. Kobayashi, Chemtech 2 (1999) 22.

[22] J. Kresta, A. Tkac, R. Prikryl, L. Malik, Macromol. Chem. 176 (1975) 157.

[23] F.J. Viersen, G. Challa, J. Reedijk, Polymer 31 (1990) 1361.

[24] P.J. Baesjou, W.L. Driessen, G. Challa, J. Reedijk, J. Mol. Catal. A: Chem. 110 (1996) 195.

[25] P.J. Baesjou, W.L. Driessen, G. Challa, J. Reedijk, J. Am. Chem. Soc. 119 (1997) 12590.

[26] P.J. Baesjou, W.L. Driessen, G. Challa, J. Reedijk, J. Mol. Catal. A: Chem. 135 (1998) 273.

[27] P.J. Baesjou, W.L. Driessen, G. Challa, J. Reedijk, J. Mol. Catal. A 140 (1999) 241.

[28] A.H.J. Tullemans, E. Bouwman, R.A.G.D. Graaff, W.L. Driessen, J. Reedijk, Recl. Trav. Chim. Pays-Bas 109 (1990) 70.

[29] E.M.D. Keegstra, J.W. Zwikker, M.R. Roest, L.W. Jenneskens, J. Org. Chem. 57 (1992) 6678

[30] P.T. Beurskens, G. Admiraal, G. Beurskens, W.P. Bosman, S. Garcia-Granda, R.O. Gould, J.M.M. Smits, C. Smykalla, The DIRDIF-97 Program System, Technical Report of the Crystallography Laboratory, University of Nijmegen, The Netherlands, 1997.

[31] G.M. Sheldrick, SHELXs-97, Program for Crystal Structure Solution, University of Göttingen, Germany, 1997.

[32] A.L. Spek, Platon, a Multipurpose Crystallographic Tool, Utrecht University, The Netherlands, 2002.

[33] G. Wilkinson, in: G. Wilkinson (Ed.), Comprehensive Coordination Chemistry, Pergamon Press, Toronto, 1987.

[34] A.W. Addison, T.N. Rao, J. Reedijk, J. van Rijn, G.C. Verschoor, J. Chem. Soc., Dalton Trans. (1984) 1349.

[35] P.E. Jurek, A.E. Martell, R.J. Motekaitis, R.D. Hancock, Inorg. Chem. 34 (1995) 1823

[36] J. Bol, Ph.D. Thesis, Leiden University, 1997.

[37] P.J. Baesjou, W.L. Driessen, J. Reedijk, A.L. Spek, Inorg. Chim. Acta 306 (2000) 237.

[38] P. Gamez, C. Simons, R.H. Steensma, W.L. Driessen, G. Challa, J. Reedijk, Eur. Polym. J. 37 (2001) 1293.

[39] W.L. Driessen, P.J. Baesjou, J.E. Bol, H. Kooijman, A.L. Spek, J. Reedijk, Inorg. Chim. Acta 324 (2001) 16.

[40] P.J. Baesjou, Ph.D. Thesis, Leiden University, 1997. 